\title{
Chemical abundances of giant stars in NGC 5053 and NGC 5634, two globular clusters associated with the Sagittarius dwarf

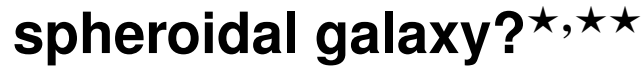

L. Sbordone ${ }^{1,2}$, L. Monaco ${ }^{3,4}$, C. Moni Bidin ${ }^{5}$, P. Bonifacio ${ }^{6}$, S. Villanova ${ }^{7}$, M. Bellazzini ${ }^{8}$, R. Ibata ${ }^{9}$, M. Chiba $^{10}$, D. Geisler ${ }^{7}$, E. Caffau ${ }^{6}$, and S. Duffau ${ }^{1,2}$

1 Millennium Institute for Astrophysics, Chile

e-mail: 1sbordon@astro.puc.cl

2 Pontificia Universidad Católica de Chile, Vicuña Mackenna 4860, Macul, Santiago, Chile

3 European Southern Observatory, Alonso de Cordova 3107, Casilla 19001, Santiago 19, Chile

4 Departamento de Ciencias Fisicas, Universidad Andres Bello, Republica 220, Santiago, Chile

5 Instituto de Astronomía, Universidad Católica del Norte, Av. Angamos 0610, Antofagasta, Chile

6 GEPI, Observatoire de Paris, PSL Resarch University, CNRS, Univ. Paris Diderot, Sorbonne Paris Cité, Place Jules Janssen, 92195 Meudon, France

7 Departamento de Astronomía, Universidad de Concepción, Casilla 160-C, Concepción, Chile

8 INAF-Osservatorio Astronomico di Bologna, via Ranzani 1, 40127 Bologna, Italy

9 Observatoire Astronomique de Strasbourg, Université de Strasbourg, CNRS, 11 rue de l'Université, 67000 Strasbourg, France

10 Astronomical Institute, Tohoku University, Aoba-ku, 980-8578 Sendai, Japan

Received 12 December 2014 / Accepted 6 May 2015

\begin{abstract}
Context. The tidal disruption of the Sagittarius dwarf spheroidal galaxy ( $\mathrm{Sgr} \mathrm{dSph}$ ) is producing the most prominent substructure in the Milky Way (MW) halo, the Sagittarius Stream. Aside from field stars, it is suspected that the Sgr dSph has lost a number of globular clusters (GC). Many Galactic GC are thought to have originated in the Sgr dSph. While for some candidates an origin in the Sgr dSph has been confirmed owing to chemical similarities, others exist whose chemical composition has never been investigated. Aims. NGC 5053 and NGC 5634 are two of these scarcely studied Sgr dSph candidate-member clusters. To characterize their composition we analyzed one giant star in NGC 5053, and two in NGC 5634.

Methods. We analyze high-resolution and signal-to-noise spectra by means of the MyGIsFOS code, determining atmospheric parameters and abundances for up to 21 species between $\mathrm{O}$ and Eu. The abundances are compared with those of MW halo field stars, of unassociated MW halo globulars, and of the metal-poor Sgr dSph main body population.

Results. We derive a metallicity of $[\mathrm{Fe} \mathrm{II} / \mathrm{H}]=-2.26 \pm 0.10$ for NGC 5053, and of $[\mathrm{Fe} \mathrm{I} / \mathrm{H}]=-1.99 \pm 0.075$ and $-1.97 \pm 0.076$ for the two stars in NGC 5634. This makes NGC 5053 one of the most metal-poor globular clusters in the MW. Both clusters display an $\alpha$ enhancement similar to the one of the halo at comparable metallicity. The two stars in NGC 5634 clearly display the Na-O anticorrelation widespread among MW globulars. Most other abundances are in good agreement with standard MW halo trends.

Conclusions. The chemistry of the Sgr dSph main body populations is similar to that of the halo at low metallicity. It is thus difficult to discriminate between an origin of NGC 5053 and NGC 5634 in the Sgr dSph, and one in the MW. However, the abundances of these clusters do appear closer to that of Sgr dSph than of the halo, favoring an origin in the Sgr dSph system.
\end{abstract}

Key words. Galaxy: abundances - globular clusters: individual: NGC 5053 - globular clusters: individual: NGC 5634 galaxies: active - stars: abundances - galaxies: individual: Sgr dSph

\section{Introduction}

It is a fundamental prediction of models of galaxy formation based on the cold dark matter (CDM) scenario, that dark matter haloes of the size of that of the Milky Way grow through the accretion of smaller subsystems (see e.g. Font et al. 2011, and references therein). These resemble very much the "protogalactic fragments" invoked by Searle \& Zinn (1978). The merging of minor systems is supposed to be a common event

\footnotetext{
* Appendix A is available in electronic form at http://www . aanda.org

$\star \star$ Atomic data are only available at the CDS via anonymous ftp to cdsarc.u-strasbg.fr (130.79.128.5) or via

http://cdsarc.u-strasbg.fr/viz-bin/qcat?J/A+A/vol/A104
}

in the early stages of the galactic history, playing a role even in the formation of the stellar disk (e.g. Lake 1989; Abadi et al. 2003). Despite this general agreement, the processes governing galaxy formation still present many obscure aspects, and understanding them is one of the greatest challenges of modern astrophysics. For example, it has been noticed that the chemical abundance patterns of present-day dwarf spheroidal (dSph) galaxies in the Local Group are very different from those observed among stars in the Galactic halo (see Vladilo et al. 2003; Venn et al. 2004, and references therein). Most noticeably, dSphs typically show a disappearance of $\alpha$ enhancement at lower metallicity than stars in the Milky Way, which is considered evidence of a slow, or bursting, star formation history. This is at variance with the properties of the stars belonging to the old, spheroidal 
Galactic component. This clearly excludes that the known dSph can represent the typical building blocks of larger structures such as the Galactic halo (Geisler et al. 2007). The observed differences are not unexpected, however, because dSphs represent a very different environment for star formation (Lanfranchi $\&$ Matteucci 2003, 2004). In any case, the observed dSphs are evolved structures that survived merging, while the models suggest that, although accretion events take place even today, the majority of the merging processes occurred very early in the history of our Galaxy. The chemical peculiarities of the present-day small satellite galaxies could have appeared later in their evolution, and the genuine building blocks could therefore have been chemically very different from what is observed today, but more similar to the resulting merged structures. The model of Font et al. (2006) implies that the satellites that formed the halo were accreted eight to nine Gyr ago, while the presently observed satellites were accreted only four to five Gyr ago, or are still being accreted.

The Sagittarius (Sgr dSph) galaxy is one of the most studied systems in the Local Group because it is the nearest known dSph (Monaco et al. 2004), currently merging with the Milky Way (Ibata et al. 1994). It thus represents a unique opportunity to study in detail both the stellar population of a dSph and the merging process of a minor satellite into a larger structure. Among Local Group galaxies the Sgr dSph is certainly exceptional, first because of the high metallicity of its dominant population $([\mathrm{Fe} / \mathrm{H}] \sim-0.5)$ compared to its relatively low luminosity $\left(M_{V}=-13.4\right.$, Mateo 1998). While the other galaxies of the Local Group follow a well-defined metallicity-luminosity relation, the Sgr dSph is clearly underluminous by almost three magnitudes with respect to this relation (see Fig. 5 of Bonifacio 2005). The chemical composition of the Sgr dSph is also very exotic because, aside from the aforementioned underabundance of $\alpha$-elements typical of small galaxies, all the other chemical elements studied so far present very peculiar patterns, clearly distinct from the Milky Way (Sbordone et al. 2007). However, this behavior is observed only for stars with $[\mathrm{Fe} / \mathrm{H}] \geq-1$. No full chemical analysis has been performed to date on field Sgr stars of lower metallicity, but the measured abundances of $\alpha$-elements suggest that the chemical differences with the Galactic halo should be much lower for $[\mathrm{Fe} / \mathrm{H}] \leq-1$ (Monaco et al. 2005). At $[\mathrm{Fe} / \mathrm{H}] \leq-1.5$ the Sgr dSph stellar population could be chemically indistinguishable from the halo, at variance with other dSphs in the Local Group (Shetrone et al. 2001, 2003), although even this difference tends to disappear at lower metallicities (Tolstoy et al. 2009).

Decades of Galactic studies have shown that crucial information about the properties and the history of a galaxy can be unveiled by the study of its globular clusters (GCs). For many aspects they can still be approximated as simple, coeval, and chemically homogeneous stellar populations, although it has been known for a while that this is not strictly true (see Gratton et al. 2012, for a review). They thus represent a snapshot of the chemical composition of the host galaxy at the time of their formation. The family of GCs associated with the Sgr dSph today counts five confirmed members. However eighteen more clusters have been proposed as belonging to the Sgr dSph (see Bellazzini et al. 2002, 2003a,b and Table 1 of Law \& Majewski 2010, hereafter L10, for a complete census). Nevertheless, the probability of a chance alignment with the Sgr streams is not negligible, and many objects in this large list of candidates are most probably not real members. In their recent analysis based on new models of the Sgr tidal disruption, L10 found that only fifteen of the candidates proposed in the literature have a non-negligible probability of belonging to the Sgr dSph. However, calculating the expected quantity of false associations in the sample, they proposed that only the nine GCs with high confidence levels most likely originate from the Sgr galaxy (in good quantitative agreement with the previous analysis by Bellazzini et al. 2003b). This sample of objects with very high membership probability includes all five of the confirmed clusters (M 54, Terzan 7, Terzan 8, Arp 2, and Palomar 12), plus Berkeley 29 (Carraro \& Bensby 2009), Whiting 1 (Carraro et al. 2007), NGC 5053, and NGC 5634 (Bellazzini et al. 2003b). The large list of GC candidate members is particularly interesting because the estimated total luminosity of the Sgr galaxy is comparable to that of Fornax (van den Bergh 2000; Majewski et al. 2003) which, with its five confirmed GCs, is known for its anomalously high GC specific frequency (van den Bergh 1998). Hence, if more than five GCs were confirmed members of the Sgr family, the parent $\mathrm{dSph}$ would be even more anomalous than Fornax, unless its total luminosity has been largely underestimated. Estimating Sgr dSph mass is, however, difficult because the galaxy is being tidally destroyed, and its relatively fast chemical evolution and presence of young, metal-rich populations hint at a very massive progenitor (Bonifacio et al. 2004; Sbordone et al. 2007; Siegel et al. 2007; Tolstoy et al. 2009; de Boer et al. 2014).

Stimulated by the results of L10, we performed a chemical analysis of NGC 5053 and NGC 5634, since no high-resolution study of the clusters abundances exists to date. These objects are particularly interesting because of their very low metallicity $([\mathrm{Fe} / \mathrm{H}] \approx-2$, Harris 1996, 2010 web version), which means that they can be used to trace the early stages of the chemical evolution of the host galaxy. In fact, NGC 5053 could be one of the most metal-poor GCs in the Sgr dSph family, and is also regarded as one of the most metal-poor GC known in the Milky Way. Law \& Majewski (2010) associate both clusters with the primary wrap of the trailing arm of the galaxy, in a section of the tail probably lost by the Sgr dSph between 3 and 5 Gyr ago. Their calculations, based on their Sgr dSph merging model and the cluster position, distance, and radial velocity, indicate that NGC 5634 has a very high probability of originating from the Sgr dSph (99.6\%), while for NGC 5053 this value is lower but still very significant $(96 \%)$.

\section{Observations and data reduction}

A high-resolution spectrum of one red giant star in NGC 5053 was retrieved with its calibration files from the Keck Observatory archive ${ }^{1}$. It was collected with one 1800s integration with HIRES (Vogt et al. 1994) on 2003 June 23 (program ID: $\mathrm{C} 02 \mathrm{H}, \mathrm{PI}$ : I. Ivans). The spectrum covered the range $438-678 \mathrm{~nm}$ at a resolution of $R=48000$, resulting from the use of a 0.'86-wide slit. The target corresponds to object 69 in the list of standard stars compiled by Stetson (2000), and its coordinates and photometric data are given in Table 1. The spectrum was reduced with HIRES Redux, the IDL-based data reduction pipeline written by Jason X. Prochaska ${ }^{2}$, for a typical signal-to-noise ratio $(\mathrm{S} / \mathrm{N})$ per pixel of about 80 . The radial velocity (RV) of the target was measured with the fxcor $\mathrm{IRAF}^{3}$

\footnotetext{
https://koa.ipac.caltech.edu/cgi-bin/KOA/ nph-KOAlogin

2 http://www.ucolick.org/ xavier/HIRedux/index.html

3 IRAF is distributed by the National Optical Astronomy Observatories, which are operated by the Association of Universities for Research in Astronomy, Inc., under cooperative agreement with the National Science Foundation.
} 
Table 1. Coordinates, photometry, heliocentric radial velocities, and determined atmospheric parameters for the three targets.

\begin{tabular}{|c|c|c|c|c|c|c|c|c|c|}
\hline Star & $\begin{array}{l}\text { RA } \\
(\mathrm{J} 2000)\end{array}$ & $\begin{array}{l}\text { Dec } \\
(\mathrm{J} 2000)\end{array}$ & $\begin{array}{c}V \\
(\mathrm{mag})\end{array}$ & $\begin{array}{c}(V-I) \\
(\mathrm{mag})\end{array}$ & $\begin{array}{r}V_{\text {helio }} \\
\mathrm{km} \mathrm{s}^{-1}\end{array}$ & $\begin{array}{r}T_{\text {eff }} \\
\mathrm{K}\end{array}$ & $\begin{array}{c}\log g \\
\mathrm{~cm} \mathrm{~s}^{-1}\end{array}$ & $\begin{array}{c}V_{\text {turb }} \\
\mathrm{km} \mathrm{s}^{-1}\end{array}$ & $\begin{array}{c}{[\mathrm{Fe} / \mathrm{H}]} \\
\operatorname{dex}\end{array}$ \\
\hline NGC 5053-69 & 13:16:35.96 & $+17: 41: 12.8$ & 14.565 & 1.163 & 42.2 & 4450 & 1.15 & 1.85 & $-2.26^{a}$ \\
\hline NGC 5634-2 & $14: 29: 30.06$ & $-05: 58: 39.4$ & 14.776 & 1.481 & -12.8 & 4085 & 0.22 & 1.72 & -1.99 \\
\hline NGC 5634-3 & $14: 29: 40.50$ & $-05: 57: 09.7$ & 14.761 & 1.432 & -20.6 & 4097 & 0.45 & 1.61 & -1.92 \\
\hline
\end{tabular}

Notes. Photometric parameters are used for NGC 5053-69, spectroscopic ones for the two stars in NGC 5634 (see Sect. 3.3). ${ }^{(a)}$ Using [Fe II/H] for this star.

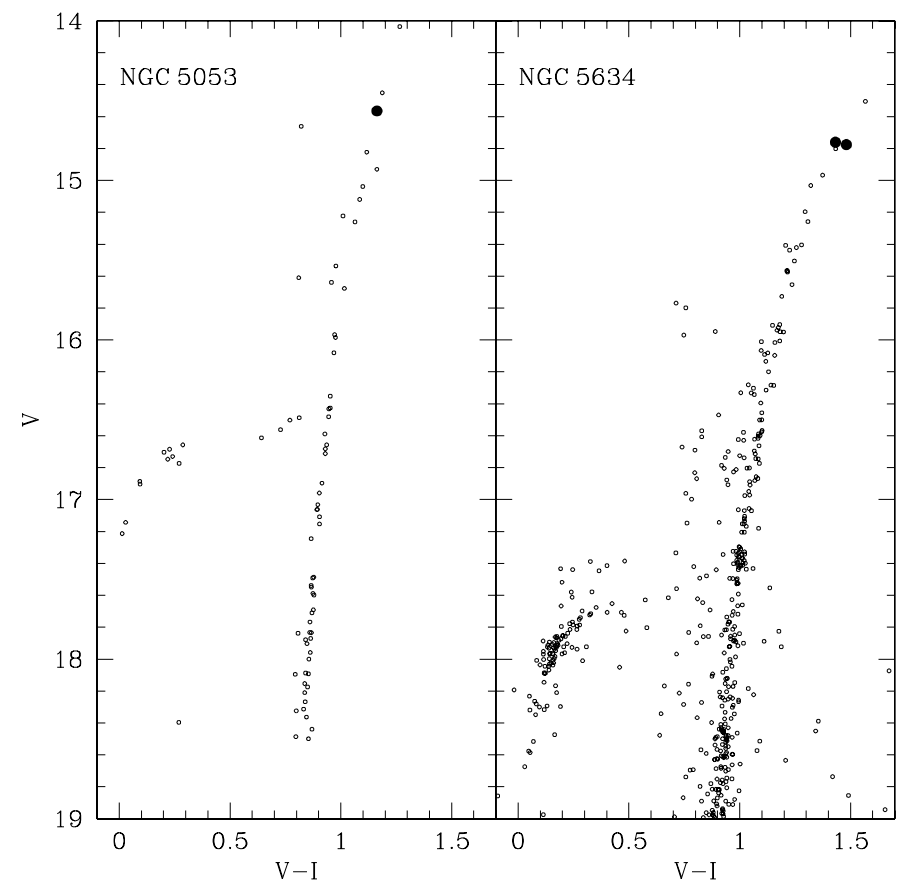

Fig. 1. Color-magnitude diagrams of the target clusters (small open circles). The large filled dots indicate the position of the program stars.

task, cross-correlating (Tonry \& Davis 1979) its spectrum with a synthetic template of a metal-poor red giant drawn from the library of Coelho et al. (2005). The observed velocity, reduced to heliocentric value was $42.2 \pm 0.7 \mathrm{~km} \mathrm{~s}^{-1}$. This value matches the systemic cluster RV proposed by Pryor \& Meylan (1993) and Geisler et al. (1995), who measured $42.8 \pm 0.3 \mathrm{~km} \mathrm{~s}^{-1}$ and $42.4 \pm 1.2 \mathrm{~km} \mathrm{~s}^{-1}$, respectively. The simultaneous coincidence of the RV, metallicity (see Sect. 4.2), and photometry (i.e. distance, Fig. 1) with the cluster values confirms that the target is a cluster member.

Two bright red giant stars in NGC 5634 were spectroscopically observed at the Subaru telescope on 2009 March 3 with the High Dispersion Spectrograph (HDS, Noguchi et al. 2002) in Echelle mode (program ID: S09A-026). The targets were selected from the photometry of Bellazzini et al. (2002, hereafter B02), and their ID numbers, coordinates, and magnitudes are given in Table 1. Four exposures were collected for star \#3 and two for star \#2, for a total integration time of three and two hours, respectively. The standard StdYb setup, $2 \times 2$ CCD binning, and the $1^{\prime \prime} .2$ slit produced high-resolution spectra $(R=$ $30000)$ in the range $410-685 \mathrm{~nm}$, secured on the blue and red CCDs simultaneously. Data were reduced as in Monaco et al. (2011) through a combination of standard IRAF tasks, and dedicated scripts are available from the HDS website ${ }^{4}$. The spectra of each star were then shifted to laboratory wavelengths and merged. The final combined spectrum of NGC 5634-2 had $S / N \approx 120$ per pixel at $600 \mathrm{~nm}$, while the results were of lesser quality for NGC $5634-3(S / N \approx 80$ per pixel) as a consequence of shorter exposure times.

The RV of the two stars was measured on each extracted spectrum with the same procedure used for NGC 5053-069, separately for the blue and the red CCD. We thus obtained eight measurements for NGC 5634-3 and four for NGC 5634-2. For each star, velocities differ by no more than $0.9 \mathrm{~km} \mathrm{~s}^{-1}$ and have a dispersion of $0.4 \mathrm{~km} \mathrm{~s}^{-1}$. The latter value will be assumed as the internal error associated with our estimates, given by the average of the single measurements: $-12.8 \pm 0.4$ and $-20.6 \pm 0.4 \mathrm{~km} \mathrm{~s}^{-1}$ for NGC 5634-2 and NGC 5634-3, respectively. This velocity difference is compatible with both stars being cluster members. The velocity dispersion of this cluster is not known. If we assume the velocities of the two stars are consistent within $1 \sigma$ this implies an estimate of $\sigma=3.9 \mathrm{~km} \mathrm{~s}^{-1}$ that is compatible with what is found in several globular clusters of similar mass. The two stars could be compatible to less than $1 \sigma$ and the velocity dispersion would be even higher. The estimates of the cluster RV in the literature are scarce, and affected by large errors. Early investigations by Mayall (1946) and Hesser et al. (1986) obtained large negative values that do not agree with our results $(-63 \pm 12$ and $-41 \pm 9 \mathrm{~km} \mathrm{~s}^{-1}$, respectively). On the contrary, our measurements agree better with Peterson (1985), who measured the $\mathrm{RV}$ of five cluster stars with an uncertainty of $25 \mathrm{~km} \mathrm{~s}^{-1}$ : their average value is $-26.0 \mathrm{~km} \mathrm{~s}^{-1}$ with an rms of $29.1 \mathrm{~km} \mathrm{~s}^{-1}$, and the resulting statistical error on the mean is $13.0 \mathrm{~km} \mathrm{~s}^{-1}$. Our targets lie very close on the cluster isochrone, have identical parameters (i.e. the same distance) and metallicity, and similar RV, hence their cluster membership is extremely likely. We conclude that the cluster RV was probably underestimated in the literature, and the average value of $-45.1 \mathrm{~km} \mathrm{~s}^{-1}$ quoted by Harris (1996) should be revised upward. This RV was assumed by L10 to assess the probability of association with the Sgr galaxy, and it matched very well the expected value for the Sgr trailing arm. As a consequence, the association of NGC 5634 with this stellar stream would be less likely after the revision of the cluster RV. However, Fig. 6 of L10 shows that this would still fall in the range predicted by the model even if increased by $\sim 25 \mathrm{~km} \mathrm{~s}^{-1}$, hence this correction is still compatible with their assignment.

The position of the program stars in the color-magnitude diagram (CMD) of the parent cluster is shown in Fig. 1, where the photometric catalogs of Stetson (2000) and B02 were used for NGC 5053 and NGC 6534, respectively. In Fig. 2 we show a portion of the resulting spectra of the three stars. Target coordinates,

\footnotetext{
4 http://www .naoj.org/Observing/Instruments/HDS/ hdsql-e.html
} 


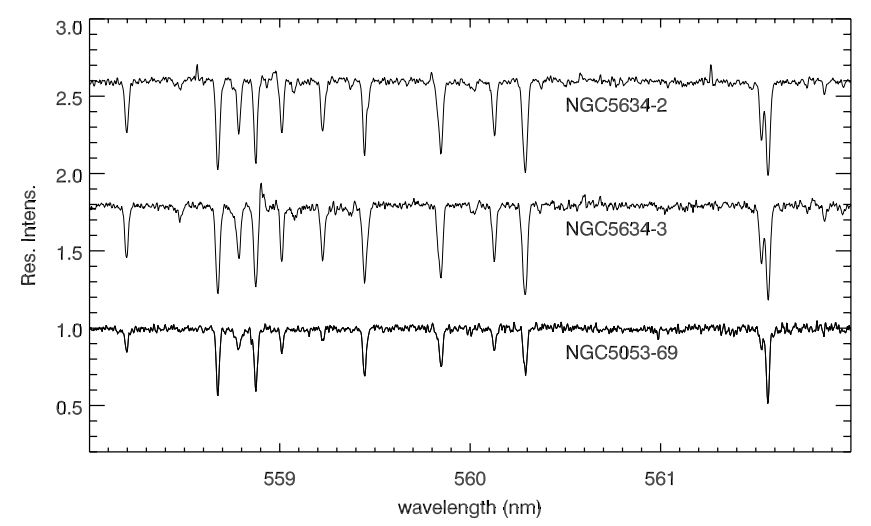

Fig. 2. A sample of the spectra for the three targets around $560 \mathrm{~nm}$. Spectra have been normalized and then vertically shifted for legibility.

photometry, radial velocities, and determined atmospheric parameters are listed in Table 1.

\section{Stellar parameters and abundance analysis}

\subsection{Spectroscopically determined parameters}

The chemical analysis was performed by means of MyGIsFOS 5 (Sbordone et al. 2014). For this purpose, a grid of synthetic spectra covering the range between $480 \mathrm{~nm}$ and $690 \mathrm{~nm}$ was computed with the following characteristics (start value, end value, step, unit): $T_{\text {eff }}(4000,5200,200, \mathrm{~K}), \log g(0.5,3$, $\left.0.5, \mathrm{~cm} \mathrm{~s}^{-1}\right) ; V_{\text {turb }}\left(1.0,3.0,1.0, \mathrm{~km} \mathrm{~s}^{-1}\right) ;[\mathrm{Fe} / \mathrm{H}](-4.0,-0.5$, $0.5, \mathrm{dex}) ;[\alpha / \mathrm{Fe}](-0.4,0.4,0.4, \mathrm{dex})$. This corresponds to a grid of 3024 atmosphere models, the majority of which belonged to the MPG grid described in Sbordone et al. (2014), with the exception of the ones with $T_{\text {eff }}=4000 \mathrm{~K}$ and $\log g=0.5$, which were computed for this work. The models were computed assuming mono-dimensional, plane-parallel, and local thermodynamical equilibrium (LTE) approximations, using ATLAS 12 (Kurucz 2005; Sbordone et al. 2004; Sbordone 2005). Synthetic spectra were then computed by means of SYNTHE (Castelli 2005). Atomic and molecular line data were retrieved from the Kurucz website, but the $\log g f$ for the lines used in the analysis were updated according to the values provided in the second version of the Gaia-ESO (GES) "clean" line list. In the grid, $\alpha$ enhancement is modeled by varying in lockstep even atomic number elements between $\mathrm{O}$ and $\mathrm{Ti}$, inclusive.

The good quality and extensive coverage of the spectra provided enough lines for a fully spectroscopic parameter determination. As discussed in more detail in Sbordone et al. (2004), MyGIsFOS determines effective temperature by searching the zero of the $T_{\text {eff }}$-LEAS ${ }^{6}$ relation, the microturbulent velocity by eliminating the dependence of $\mathrm{Fe}$ I abundance from line equivalent width, the surface gravity by imposing Fe I-Fe II ionization equilibrium. Since $[\alpha / \mathrm{Fe}]$ may have a significant influence on the atmospheric structure and affect line formation, a "global" $[\alpha / \mathrm{Fe}]$ is one of the dimensions of the synthetic grid. The synthetic grid needs to be broadened to match the combination of instrumental and macroturbulent/rotational broadening for each star. After checking the fit profiles on a number of unblended, metallic lines, we applied a Gaussian broadening to the grid of $F W H M=9 \mathrm{~km} \mathrm{~s}^{-1}$ for all three stars.

\footnotetext{
5 mygisfos.obspm.fr

6 Lower Energy Abundance Slope, see Sbordone et al. (2014).
}

\subsection{Photometric parameters estimates}

In addition to the fully spectroscopic parameter estimates, we also estimated $T_{\text {eff }}$ from the de-reddened color $(V-I)$ through the equations of Alonso et al. $(1999,2001)$, assuming $E(V-$ $I)=0.08$ mag for NGC 5634 (Bellazzini et al. 2002), and $E(B-V)=0.017 \mathrm{mag}$ for NGC 5053 (Nemec 2004), with $E(V-I)=1.34 \times E(B-V)($ Dean et al. 1978). The adopted color-temperature relation is independent of metallicity.

The surface gravity was calculated through the equation

$\log g=\log M+4 \log T_{\text {eff }}+0.4\left(M_{\mathrm{V}}+\mathrm{BC}\right)-12.503$,

obtained from basic relations, where $M$ is the mass, $\mathrm{BC}$ is the bolometric correction, and the solar values $T_{\odot}=5777 \mathrm{~K}$, $\log g_{\odot}=4.44, M_{\mathrm{bol}, \odot}=4.75$ are assumed in the calculation of the constant term. The stellar mass was fixed to $0.80 \pm 0.05 M_{\odot}$ for all the stars, and $\mathrm{BC}$ was deduced from the temperature interpolating the tables of Alonso et al. (1999). The absolute magnitude in the $V$ band was estimated from the cluster reddening, defined as before, and distance modulus $(m-M)_{V}=16.23$ (Harris 1996) and 17.36 mag (Bellazzini et al. 2002) for NGC 5053 and NGC 5634, respectively. Starting from these values for $T_{\text {eff }}$ and $\log g$, we re-derived $V_{\text {turb }}$ and abundances as we did for the fully spectroscopic case: for brevity, we will refer to this set of parameters as "photometric" parameters from now on.

The temperature of the two stars in NGC 5634 are very similar to the spectroscopic values $\left(\Delta T_{\text {eff }}<40 \mathrm{~K}\right)$, and the gravities are higher but still compatible within uncertainties. The photometric gravity of NGC 5053-69 is, on the contrary, 0.9 dex higher than the spectroscopic value. In particular, the spectroscopic parameters of this star cannot satisfy the basic relation above, unless one or more of the other input quantities (cluster parameters, stellar mass, BC) are revised to unrealistic values.

\subsection{Choice of adopted atmospheric parameters}

The discrepancy between photometric and spectroscopic atmospheric parameters is a well-documented fact for giant stars around or below $[\mathrm{Fe} / \mathrm{H}]=-2$. In two recent studies, for instance, Mashonkina et al. (2011) and Bergemann et al. (2012) study the case of the metal-poor giant HD 122563, whose parameters can be reliably derived from photometry and HIPPARCOSbased parallaxes. Said parameters $\left(T_{\text {eff }}=4665 \mathrm{~K}, \log g=1.64\right.$, $\left.V_{\text {turb }}=1.61 \mathrm{~km} \mathrm{~s}^{-1},[\mathrm{Fe} / \mathrm{H}]=-2.60\right)$ are quite close to the ones photometrically derived for NGC 5053-69, and, when using them in a 1D-LTE analysis, HD 122563 shows a Fe I-Fe II imbalance and residual LEAS quite close to the ones NGC 5053-69 displays in the present study. Mashonkina et al. (2011) manages to recover a satisfactory ionization balance for HD 122563 when treating $\mathrm{Fe}$ I line formation taking into account departures from LTE (NLTE), but is left with an unacceptable LEAS, albeit a reduced one with respect to the LTE case. Bergemann et al. (2012) also employs NLTE line formation for Fe I, but in association with horizontally averaged 3D hydrodynamical models, which preserve the vertical temperature structure of 3D models. Despite this further refinement, the LEAS problem of HD 122563 remains unsolved, likely indicating that the horizontal temperature variations (that are averaged out in the Bergemann et al. 2012 models) must be accounted for together with NLTE to properly describe Fe I line formation in these cool, metal-poor giants. It is thus evident that NLTE affects Fe I line formation in a relevant way in stars like NGC 5053-69, and likely, to a lower extent, NGC 5634-2 and NGC 5634-3. 


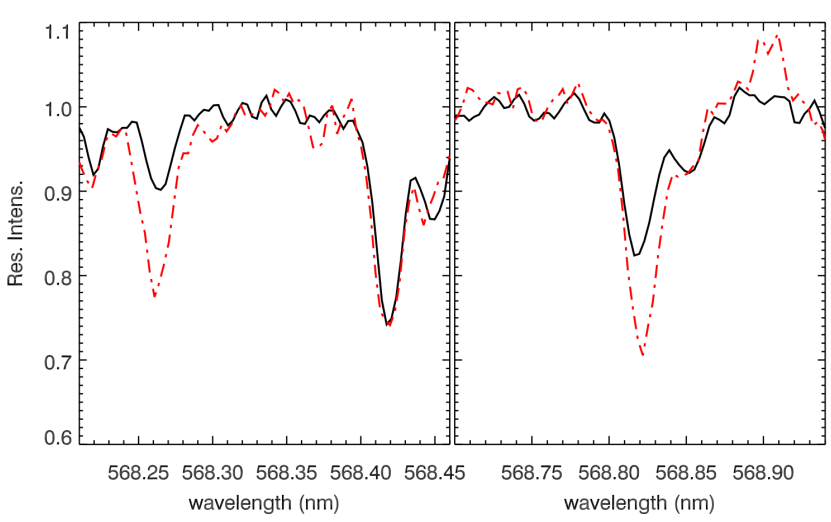

Fig. 3. Left panel: NaI $568.2 \mathrm{~nm}$ line and Sc II $568.4 \mathrm{~nm}$ lines in the spectra of stars NGC 5634-2 (black solid line) and NGC 5634-3 (red dash-dotted line). The MyGIsFOS fit to this specific feature is $[\mathrm{Na} / \mathrm{H}]=-2.22$ for NGC 5634-2, and -1.72 for NGC 5634-3. Right panel: NaI $568.8 \mathrm{~nm}$ line in the same stars. Abundances here are $[\mathrm{Na} / \mathrm{H}]=-2.15$ and -1.76 . The remarkable difference in $\mathrm{Na}$ abundance between the two stars is quite evident.

In the case of NGC 5053-69, the LTE treatment significantly underestimates Fe ionization and upper-level populations (Mashonkina et al. 2011), leading to an overestimate of Fe I line strength, and a consequent underestimate of the derived abundance. We thus decided to adopt the photometric set of parameters for NGC 5053-69, employ Fe II for the determination of $[\mathrm{Fe} / \mathrm{H}]$, and derive all the $[\mathrm{X} / \mathrm{Fe}]$ ratios with respect to $[\mathrm{Fe} \mathrm{II} / \mathrm{H}]$.

The situation is less straightforward in the case of the two stars in NGC 5634. The two stars show quasi-identical spectra, which the spectroscopic parameter estimate underscores by providing practically equal parameters. Despite this agreement, they show a significant $(V-I)$ difference, which translates to a $86 \mathrm{~K}$ photometric $T_{\text {eff }}$ difference. Most likely, this is a consequence of NGC 5634-3 being a second-generation star, as clearly indicated by its $\mathrm{Na}$ abundance (see Sect. 4 and Fig. 3). The photometric effect of abundance variations in globular clusters was studied in detail in Sbordone et al. (2011), where it can be seen that second-generation stars in the upper RGB become slightly bluer than first-generation ones, essentially because of the flux lost in the UV due to stronger $\mathrm{NH}$ absorption being transferred more in the $V$ band than in the $I$. An inspection of Sbordone et al. (2011) "reference" and "CNONa2" isochrones around the spectroscopic temperature of NGC 5634-3 shows an effect of about 0.04 mag in $(V-I)$, strikingly similar to the difference in color between NGC 5634-2 and NGC 5634-3. This cannot be taken strictly at face value: the Sbordone et al. (2011) calculations were performed at a slightly higher metallicity $([\mathrm{Fe} / \mathrm{H}]=-1.62)$, and the assumed variations of $\mathrm{C}, \mathrm{N}$, and $\mathrm{O}$ abundances were likely larger than the ones encountered in NGC 5634. However, it is a clear indication that, in the absence of calibrations computed taking into account abundance variations such as the one encountered in globular clusters, photometric temperature estimates might be significantly skewed in second-generation stars in the upper part of the RGB. It is interesting to note how star NGC 5634-2, on the other hand, shows an excellent agreement between photometric and spectroscopic temperature, and a photometric gravity that is only 0.33 dex higher than the spectroscopic. Although we are considering one single star here, this hints at the fact that NLTE effects on Fe I on $T_{\text {eff }}$ and gravity are strongly reduced at the metallicity of NGC 5634. For this reason, we feel confident that spectroscopic parameters can be employed in this case, and we consider them preferable owing to their insensitivity to the effects of $\mathrm{CNO}$ abundance variations.

In Table 3 we list the variations in $[\mathrm{X} / \mathrm{H}]$ in each star that would have originated from employing the "rejected" set of parameters for the star, i.e. the photometric parameters for the two stars in NGC 5634, and the spectroscopic ones in NGC 5053-69. Here, the strongest variations are, as expected, encountered in the ionized species in NGC 5053-69. Since the rejected parameter set would force gravity to recover Fe I-Fe II ionization equilibrium, abundances for Fe II and other ionized species decrease consistently by $0.25-0.35$ dex. Most neutral species decrease of about 0.05 dex (likely due to the roughly $100 \mathrm{~K}$ lower $T_{\text {eff }}$ in the rejected set), with the exception of $\mathrm{Zn}$, which shows a stronger effect. The effect is the opposite in NGC 5634-2, where employing the rejected photometric set would break Fe ionization equilibrium, raising the abundance of ionized species, although, in this case, Fe II varies more than most of the other ionized elements. Also affected is O I, since the [O I] $630 \mathrm{~nm}$ line is, naturally, sensitive to pressure. Finally, NGC 5634-3 has very close photometric and spectroscopic parameters, leading to almost identical abundances. As usually observed in these cases, $[\mathrm{X} / \mathrm{Fe}]$ abundance ratios produced taking care of matching ionization stages are quite robust against variations in atmospheric parameters.

\subsection{Detailed abundances}

After parameter determination, MyGIsFOS derived detailed abundances for all the elements for which regions had been provided and viable lines were found. All the employed regions, as well as the observed and best-fitting synthetic profiles, can be obtained online via Vizier (see Appendix A). Hyper fine splitting (HFS) values for the used lines were instead derived from the fourth version of the GES line list (Heiter et al., in prep.), and used for the lines of Sc II, V I, Mn I, Co I, Cu I, Ba II, and Eu II. Isotopic mixture for $\mathrm{Eu}$ was derived from Anders \& Grevesse (1989).

Abundances for all elements were measured within MyGIsFOS, with the exception of oxygen, whose abundance was determined in NGC 5634 by measuring the [OI] $630.0304 \mathrm{~nm}$ line. The line could be measured by MyGIsFOS in NGC 5634-2, but not in NGC 5634-3 since the line was contaminated by telluric absorptions. In the latter case, the region around the [O I] $630.0304 \mathrm{~nm}$ line was decontaminated from telluric lines according to the following procedure. First of all, we calculated a synthetic telluric spectrum where parameters were varied in order to match the depth and width of the unblended telluric features. Then we divided the spectral region surrounding the [OI] $630.0304 \mathrm{~nm}$ line by this spectrum. We then proceeded to fit the oxygen line in both stars as follows: for each star, an ATLAS 12 model at the final parameters and abundances was computed, from which two small grids of synthetic spectra were computed in the region surrounding the oxygen line at varying $\mathrm{O}$ abundances. The fitprofile linefitting code (Thygesen et al., in prep.) was then employed to derive the best-fitting $\mathrm{O}$ abundance by $\chi^{2}$ minimization. For consistency, fitprofile was employed to derive $\mathrm{O}$ abundances in both NGC 5634-2 and NGC 5634-3.

\section{Results}

Derived abundances are in Table 2. The assumed solar abundances used in the grid are also listed in this table, and were taken from the compilation of Lodders et al. (2009), except for 
Table 2. Detailed abundances for the three targets.

\begin{tabular}{|c|c|c|c|c|c|c|c|c|c|c|c|c|c|c|c|c|}
\hline \multirow[b]{2}{*}{ Ion } & \multicolumn{5}{|c|}{ NGC 5634-2 } & \multicolumn{6}{|c|}{ NGC 5634-3 } & \multicolumn{4}{|c|}{ NGC 5053-69 } & \multirow[b]{2}{*}{$\begin{array}{r}\sigma \\
{[\mathrm{X} / \mathrm{Fe}]}\end{array}$} \\
\hline & $\begin{array}{l}\text { Solar } \\
\text { abu. }\end{array}$ & $N$ & {$[\mathrm{X} / \mathrm{H}]$} & $\begin{array}{r}\sigma \\
{[\mathrm{X} / \mathrm{H}]}\end{array}$ & {$[\mathrm{X} / \mathrm{Fe}]^{a}$} & $\begin{array}{r}\sigma \\
{[\mathrm{X} / \mathrm{Fe}]}\end{array}$ & $N$ & {$[\mathrm{X} / \mathrm{H}]$} & $\begin{array}{r}\sigma \\
{[\mathrm{X} / \mathrm{H}]}\end{array}$ & {$[\mathrm{X} / \mathrm{Fe}]^{a}$} & $\begin{array}{r}\sigma \\
{[\mathrm{X} / \mathrm{Fe}]}\end{array}$ & $N$ & {$[\mathrm{X} / \mathrm{H}]$} & $\begin{array}{r}\sigma \\
{[\mathrm{X} / \mathrm{H}]}\end{array}$ & {$[\mathrm{X} / \mathrm{Fe}]^{b}$} & \\
\hline O I & 8.76 & 1 & -1.47 & - & 0.56 & - & 1 & -1.63 & - & 0.29 & - & - & - & - & - & - \\
\hline $\mathrm{NaI}$ & 6.30 & 2 & -2.19 & 0.05 & -0.20 & 0.09 & 3 & -1.79 & 0.09 & 0.12 & 0.12 & 1 & -2.05 & - & 0.21 & - \\
\hline Mg I & 7.54 & 1 & -1.40 & - & 0.59 & - & 1 & -1.46 & - & 0.46 & - & - & - & - & - & - \\
\hline $\mathrm{Al} \mathrm{I}$ & 6.47 & - & - & - & - & - & 1 & -1.32 & - & 0.59 & - & - & - & - & - & - \\
\hline Si I & 7.52 & 3 & -1.56 & 0.16 & 0.43 & 0.18 & 3 & -1.58 & 0.16 & 0.33 & 0.17 & - & - & - & - & - \\
\hline $\mathrm{Ca} \mathrm{I}$ & 6.33 & 12 & -1.73 & 0.11 & 0.27 & 0.13 & 9 & -1.62 & 0.08 & 0.29 & 0.11 & 9 & -2.15 & 0.08 & 0.10 & 0.13 \\
\hline Sc II & 3.10 & 10 & -1.78 & 0.09 & 0.25 & 0.18 & 8 & -1.65 & 0.06 & 0.27 & 0.16 & 9 & -2.09 & 0.13 & 0.10 & 0.17 \\
\hline Ti I & 4.90 & 10 & -1.75 & 0.05 & 0.24 & 0.09 & 9 & -1.70 & 0.10 & 0.21 & 0.13 & 3 & -2.33 & 0.13 & -0.07 & 0.17 \\
\hline Ti II & 4.90 & 2 & -1.71 & 0.09 & 0.32 & 0.18 & 2 & -1.54 & 0.13 & 0.38 & 0.20 & 3 & -2.08 & 0.10 & 0.10 & 0.14 \\
\hline V I & 4.00 & 16 & -2.19 & 0.12 & -0.20 & 0.14 & 16 & -2.09 & 0.11 & -0.18 & 0.13 & - & - & - & - & - \\
\hline Cr I & 5.64 & 3 & -2.16 & 0.05 & -0.16 & 0.09 & 2 & -2.09 & 0.16 & -0.17 & 0.17 & 2 & -2.75 & 0.05 & -0.49 & 0.12 \\
\hline Mn I & 5.37 & 5 & -2.36 & 0.15 & -0.37 & 0.16 & 5 & -2.31 & 0.14 & -0.40 & 0.16 & 1 & -2.88 & - & -0.62 & - \\
\hline $\mathrm{Fe} \mathrm{I}$ & 7.52 & 52 & -1.99 & 0.07 & - & 0.11 & 55 & -1.92 & 0.08 & - & 0.11 & 27 & -2.48 & 0.08 & -0.22 & 0.13 \\
\hline Fe II & 7.52 & 9 & -2.03 & 0.16 & - & 0.22 & 7 & -1.92 & 0.15 & - & 0.22 & 5 & -2.26 & 0.10 & - & 0.14 \\
\hline Co I & 4.92 & 4 & -1.94 & 0.07 & 0.05 & 0.10 & 3 & -1.94 & 0.07 & -0.02 & 0.10 & - & - & - & - & - \\
\hline $\mathrm{Ni} I$ & 6.23 & 7 & -2.16 & 0.15 & -0.17 & 0.17 & 6 & -2.03 & 0.15 & -0.11 & 0.17 & 1 & -2.45 & - & -0.19 & - \\
\hline $\mathrm{Cu} \mathrm{I}$ & 4.21 & - & - & - & - & - & 1 & -2.43 & - & -0.52 & - & - & - & - & - & - \\
\hline Zn I & 4.62 & 1 & -2.06 & - & -0.07 & - & 1 & -2.15 & - & -0.23 & - & 1 & -2.42 & - & -0.16 & - \\
\hline Y II & 2.21 & 4 & -2.43 & 0.14 & -0.40 & 0.21 & 4 & -2.25 & 0.13 & -0.33 & 0.20 & 2 & -2.89 & 0.10 & -0.63 & 0.14 \\
\hline Ba II & 2.17 & 1 & -2.12 & - & -0.09 & - & 1 & -1.82 & - & 0.10 & - & 3 & -2.51 & 0.07 & -0.25 & 0.12 \\
\hline Eu II & 0.52 & 1 & -1.37 & - & 0.66 & - & 1 & -1.17 & - & 0.75 & - & - & - & - & - & - \\
\hline
\end{tabular}

Notes. $N$ indicates the number of regions used. $\sigma[\mathrm{X} / \mathrm{H}]$ represents the scatter of the abundance determined from different regions, when at least two are measured for the ion. ${ }^{(a)}[\mathrm{X} / \mathrm{Fe}]$ computed against Fe I for neutral species except O I, against Fe II for ionized species and O I. ${ }^{(b)} \mathrm{All}[\mathrm{X} / \mathrm{Fe}]$ computed against Fe II.

Table 3. Variation in $[\mathrm{X} / \mathrm{H}]$ when using for each star the alternate parameter set with respect to the one chosen.

\begin{tabular}{|c|c|c|c|}
\hline & $\begin{array}{r}\text { NGC 5053-69 } \\
T_{\text {eff }}=4343 \\
\log g=0.26 \\
V_{\text {turb }}=1.7 \\
\Delta[\mathrm{X} / \mathrm{H}]\end{array}$ & $\begin{array}{r}\text { NGC 5634-2 } \\
T_{\text {eff }}=4071 \\
\log g=0.55 \\
V_{\text {turb }}=1.7 \\
\Delta[\mathrm{X} / \mathrm{H}]\end{array}$ & $\begin{array}{r}\text { NGC 5634-3 } \\
T_{\text {eff }}=4135 \\
\log g=0.55 \\
V_{\text {turb }}=1.6 \\
\Delta[\mathrm{X} / \mathrm{H}]\end{array}$ \\
\hline OI & - & 0.19 & 0.04 \\
\hline $\mathrm{NaI}$ & -0.01 & -0.05 & 0.02 \\
\hline Mg I & - & -0.01 & 0.00 \\
\hline $\mathrm{Al} \mathrm{I}$ & - & - & 0.02 \\
\hline Si I & - & 0.06 & 0.01 \\
\hline $\mathrm{Ca}$ I & 0.02 & -0.06 & -0.01 \\
\hline Sc II & -0.37 & 0.16 & 0.03 \\
\hline Ti I & -0.07 & -0.05 & 0.04 \\
\hline Ti II & -0.26 & 0.07 & -0.02 \\
\hline V I & - & 0.04 & 0.08 \\
\hline Cr I & -0.03 & -0.05 & 0.05 \\
\hline Mn I & 0.01 & -0.04 & 0.05 \\
\hline $\mathrm{Fe} \mathrm{I}$ & -0.04 & -0.02 & 0.03 \\
\hline Fe II & -0.27 & 0.21 & 0.06 \\
\hline Co I & - & 0.02 & 0.05 \\
\hline Ni I & -0.07 & 0.00 & 0.04 \\
\hline $\mathrm{Cu} \mathrm{I}$ & - & - & 0.04 \\
\hline Zn I & -0.14 & 0.10 & -0.02 \\
\hline Y II & -0.29 & 0.07 & 0.02 \\
\hline Ba II & -0.26 & 0.12 & 0.04 \\
\hline Eu II & - & 0.17 & 0.04 \\
\hline
\end{tabular}

Notes. Variations are computed as rejected-chosen.

O, Fe, and Eu, which where taken from Caffau et al. (2011a), while Table 4 lists the impact on the derived abundances for star NGC 5634-3 of altering each atmospheric parameter by an amount roughly equivalent to the estimated error.
In Figs. 4 to 10 we plot various chemical abundance ratios in the three studied stars compared with values in different Sgr dSph populations, other globular clusters, and stars in the Milky Way disk and halo. In all these figures, typical error bars for the present analysis are overplotted: they correspond to lineto-line scatter as indicated in Table 2 . Where only one line was measured, we show a default 0.15 dex error bar for $[\mathrm{X} / \mathrm{Fe}]$, and the corresponding bar is traced in gray. For the globular clusters associated with $\mathrm{Sgr} \mathrm{dSph}$ (red symbols), average cluster values have been retrieved from Carretta et al. (2014), and come from Carretta et al. (2014) and Mottini et al. (2008) for Terzan 8, Mottini et al. (2008) for Arp 2, Carretta et al. (2010) for M 54, Cohen (2004) for Palomar 12, and Sbordone et al. (2005) for Terzan 7. Milky Way stars (gray symbols) are taken from the Venn et al. (2004) compilation, while the two MW comparison clusters NGC 6397 and NGC 5897 (cyan symbols) come from Lind et al. (2011a) and Koch \& McWilliam (2014), respectively. The data points referring to the Sgr dSph metal-poor main body populations come from a companion work to the present one, based on UVES (Dekker et al. 2000) spectra. The full analysis of these stars will be presented in Sbordone et al. (in prep.), here we just point out that these stars are similar (albeit slightly less evolved) to the ones studied here, and have been analyzed by means of MyGIsFOS, using the same grid and the same region list employed in this paper. Metal-rich $([\mathrm{Fe} / \mathrm{H}]>-1.0) \mathrm{Sgr} \mathrm{dSph}$ data points are taken from Sbordone et al. (2007).

\subsection{NGC 5634}

The two stars examined are very high-luminosity red giants, as made evident by their very low gravity, which required a slight extrapolation of the MyGIsFOS grid in the gravity dimension (Table 1). In fact, both stars show a clear emission component on $\mathrm{H} \alpha$ wings (see Fig. 11), apparently asymmetric 
Table 4. Variations in the derived abundances due to varying atmosphere parameters in star NGC 5634-3.

\begin{tabular}{|c|c|c|c|c|c|c|c|c|c|c|c|}
\hline Variation & OI & $\mathrm{NaI}$ & $\operatorname{Mg} \mathrm{I}$ & $\mathrm{Al} \mathrm{I}$ & Si I & $\mathrm{CaI}$ & Sc I & Ti I & Ti II & VI & Cr I \\
\hline$T_{\text {eff }}+50 \mathrm{~K}$ & 0.00 & 0.04 & 0.03 & 0.03 & -0.01 & 0.08 & -0.01 & 0.09 & 0.00 & 0.10 & 0.09 \\
\hline$T_{\text {eff }}-50 \mathrm{~K}$ & -0.01 & -0.04 & -0.04 & -0.03 & 0.00 & -0.06 & -0.01 & -0.09 & 0.00 & -0.10 & -0.09 \\
\hline $\log g+0.3$ & 0.10 & -0.03 & -0.01 & 0.01 & 0.05 & -0.03 & 0.13 & -0.02 & 0.06 & -0.01 & -0.02 \\
\hline $\log g-0.3$ & -0.12 & 0.03 & 0.01 & -0.01 & -0.05 & 0.05 & -0.14 & 0.02 & -0.05 & 0.01 & 0.02 \\
\hline$V_{\text {turb }}+0.2 \mathrm{~km} \mathrm{~s}^{-1}$ & -0.02 & -0.02 & -0.03 & -0.01 & -0.01 & -0.07 & -0.06 & -0.03 & -0.07 & -0.01 & -0.08 \\
\hline$V_{\text {turb }}-0.2 \mathrm{~km} \mathrm{~s}^{-1}$ & 0.00 & 0.02 & 0.03 & 0.01 & 0.01 & 0.11 & 0.06 & 0.07 & 0.08 & 0.01 & 0.11 \\
\hline$[\alpha / \mathrm{Fe}]+0.2$ & 0.01 & -0.01 & 0.00 & -0.01 & 0.04 & 0.02 & 0.04 & 0.05 & 0.05 & 0.00 & 0.00 \\
\hline$[\alpha / \mathrm{Fe}]-0.2$ & -0.07 & 0.02 & -0.01 & 0.01 & -0.04 & -0.03 & -0.05 & -0.05 & -0.05 & 0.00 & 0.00 \\
\hline Variation & Mn I & $\mathrm{Fe} \mathrm{I}$ & Fe II & CoI & $\mathrm{Ni} I$ & $\mathrm{Cu} \mathrm{I}$ & $\mathrm{ZnI}$ & Y II & Ba II & Eu II & \\
\hline$T_{\text {eff }}+50 \mathrm{~K}$ & 0.07 & 0.04 & -0.08 & 0.06 & 0.05 & 0.07 & -0.04 & 0.01 & 0.02 & -0.02 & \\
\hline$T_{\text {eff }}-50 \mathrm{~K}$ & -0.07 & -0.04 & 0.08 & -0.06 & -0.05 & -0.08 & 0.04 & -0.02 & -0.02 & 0.02 & \\
\hline $\log g+0.3$ & -0.01 & 0.00 & 0.19 & 0.03 & 0.02 & -0.03 & 0.07 & 0.08 & 0.14 & 0.19 & \\
\hline $\log g-0.3$ & 0.01 & 0.00 & -0.16 & -0.03 & -0.02 & 0.02 & -0.07 & -0.07 & -0.13 & -0.15 & \\
\hline$V_{\text {turb }}+0.2 \mathrm{~km} \mathrm{~s}^{-1}$ & -0.03 & -0.06 & -0.06 & -0.01 & -0.03 & -0.04 & -0.05 & -0.08 & -0.23 & -0.01 & \\
\hline$V_{\text {turb }}-0.2 \mathrm{~km} \mathrm{~s}^{-1}$ & 0.03 & 0.07 & 0.06 & 0.01 & 0.04 & 0.05 & 0.06 & 0.09 & 0.30 & 0.01 & \\
\hline$[\alpha / \mathrm{Fe}]+0.2$ & 0.00 & 0.00 & 0.07 & 0.01 & 0.00 & -0.02 & 0.10 & 0.03 & 0.06 & 0.10 & \\
\hline$[\alpha / \mathrm{Fe}]-0.2$ & 0.00 & -0.01 & -0.07 & -0.01 & 0.00 & 0.02 & -0.09 & -0.03 & -0.06 & -0.09 & \\
\hline
\end{tabular}

Notes. Since the parameters of the three stars are quite similar, these systematic errors can be applied to all three stars.

with respect to the absorption line, which could be interpreted as evidence of mass loss from the star. The emission appears to be more blueshifted (or more blue-asymmetric) in star NGC 5634-3, leading to a slightly different center of the absorption component.

The two stars are extremely similar, with atmospheric parameters whose differences are well within the observational uncertainties. Chemical abundances are also, in most cases, extremely similar between the two stars. The $[\mathrm{Fe} / \mathrm{H}](-1.94 \pm 0.08$ and $-1.93 \pm 0.08$ for NGC 5634-3) indicate a cluster metallicity in excellent agreement with existing photometric estimates $([\mathrm{Fe} / \mathrm{H}]=-1.94$, see Bellazzini et al. 2002, and references therein).

Oxygen appears enhanced with respect to the solar ratio in a fashion compatible with typical halo values at this metallicity in NGC 5634-2, less so in NGC 5634-3. The [O/Fe] ratio differs by 0.27 dex between the two stars, anticorrelating with the difference in $\mathrm{Na}$ abundance.

Sodium is the element that displays the most strikingly different abundance between the two stars. They differ by 0.34 dex, well beyond the line-to-line dispersion, with star NGC 5634-3 being the more Na-rich. The difference is readily visible in the spectrum, as shown in Fig. 3, where NaI features appear much stronger in NGC 5634-3, despite almost identical parameters. The clear $\mathrm{Na}$ abundance difference, together with the opposite $\mathrm{O}$ abundance difference, make it highly probable that NGC 5634 displays a significant $\mathrm{Na}$ abundance spread, and possibly a Na-O abundance anticorrelation, as is almost universally observed in globular clusters. It should be remarked that the observed $\mathrm{Na}$ abundance difference is about half of the typical full extent of the $\mathrm{Na}$ abundance spread as observed in most GCs (see for instance Fig. 2 in Gratton et al. 2012). A sample of only two stars, obviously, does not allow one to infer the full extent of the spread, nor the numerical significance of the second generation in NGC 5634.

NLTE corrections are available for two of the Na I lines we used (568.2 $\mathrm{nm}$ and $568.8 \mathrm{~nm}$ ) as derived from the calculations of Lind et al. (2011b), and made available on the web through the INSPECT ${ }^{7}$ interface. The available calculations have a lower $\log g$ limit of 1.0 , so we could not test the values for the atmospheric parameters of our stars. However, corrections appear to be very small in this parameter domain ( $0.03 \mathrm{dex}$ for the $568.2 \mathrm{~nm}$ line, and 0.05 for the $568.8 \mathrm{~nm}$ line with the parameters and line strength of star NGC 5634-2).

The $\alpha$ elements $\mathrm{Mg}, \mathrm{Si}$, and $\mathrm{Ca}$ are all enhanced with respect to iron by $\sim 0.3$ to 0.5 dex. Titanium is also enhanced by about $0.3 \mathrm{dex}$; one should note, however, that nucleosynthetically it is not a pure $\alpha$ element, since it may be synthesized in nuclear statistical equilibrium, together with iron-peak elements. The odd light element Al is only detected in star NGC 5634-3 and it is strongly enhanced over iron $(0.6 \mathrm{dex})$. If on the one hand this is not too surprising, since this star is also enhanced in $\mathrm{Na}$, it is surprising that this does not appear to be accompanied by a decrease in $\mathrm{Mg}$ abundance as is usually observed (see e.g. Gratton et al. 2001). The iron peak elements, V, Cr, Co, and Ni (Fig. 5) follow the iron abundance, while Sc seems to be slightly enhanced with respect to iron in both stars, and Mn and $\mathrm{Cu}$ slightly underabundant with respect to iron. In fact $\mathrm{Mn}$ is found to be underabundant both in Sgr dSph stars (McWilliam et al. 2003a,b; Sbordone et al. 2007) and in Milky Way stars (Gratton 1989; Cayrel et al. 2004) at low metallicity. Bergemann \& Gehren (2008) presented NLTE computations that increase the $\mathrm{Mn}$ abundances so that that $[\mathrm{Mn} / \mathrm{Fe}] \sim 0$. Copper is measured in NGC 5634-3 only, and by a single line. The fact that is underabundant with respect to iron is coherent with what is observed in Galactic stars (Mishenina et al. 2002; Bihain et al. 2004). One should note, however, that Bonifacio et al. (2010) warned against the possible effects of granulation and NLTE that may affect the determination of $\mathrm{Cu}$ abundances, based on the differences found between the abundances in dwarfs and giants in the globular cluster NGC 6397, which has a metallicity similar to NGC 5634. Zinc is slightly underabundant with respect to iron in both stars, not inconsistent with the Galactic trend at this metallicity (Mishenina et al. 2002; Bihain et al. 2004), but also not inconsistent with what is observed in Sgr dSph stars at

http://www . inspect-stars.com 


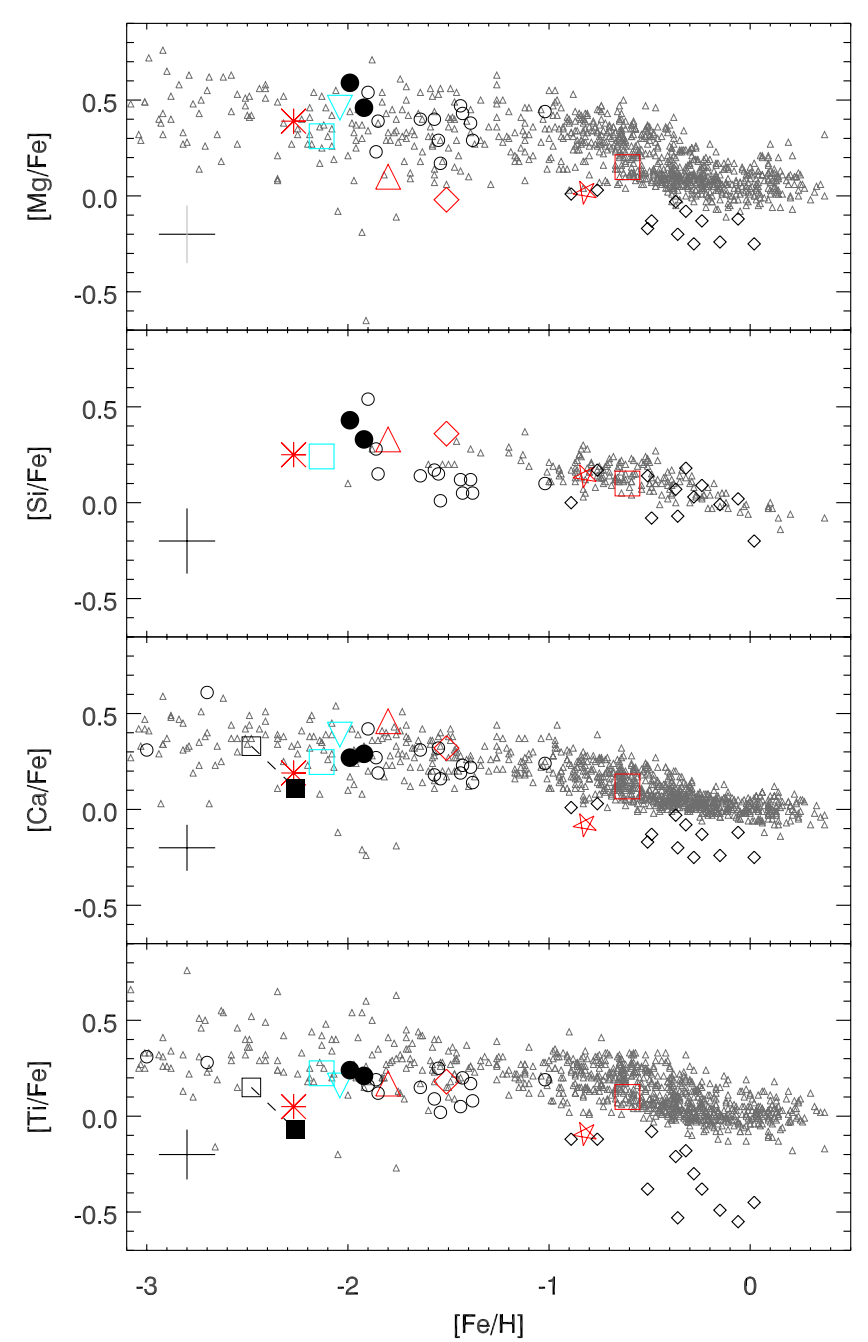

Fig. 4. $[\mathrm{Mg} \mathrm{I} / \mathrm{Fe}]$, [Si I/Fe], [Ca I/Fe], and [Ti I/Fe] ratios plotted against $[\mathrm{Fe} / \mathrm{H}]$ for the studied stars in NGC 5053 (filled black squares) and NGC 5634 (filled black circles), compared with Sgr dSph main body populations (open black circles, Sbordone et al., in prep.; open black diamonds, Sbordone et al. 2007), and mean values for five globular clusters related to Sgr dSph: Ter 8 (red asterisk), Arp 2 (open red triangle), M 54 (open red diamond), Pal 12 (open star), and Ter 7 (open red square). Small open gray triangles are Milky Way stars (Venn et al. 2004; Reddy et al. 2006). Large open cyan symbols are MW globular clusters NGC 6397 (square) and NGC 5897 (triangle). The large open black square connected by a dashed line with the filled black square represents the result for NGC 5053 when plotting $[\alpha / \mathrm{Fe} \mathrm{I}]$ vs. $[\mathrm{Fe} \mathrm{I} / \mathrm{H}]$, with the latter represented by the open symbol.

higher metallicity (Sbordone et al. 2007). The neutron capture element Y (Fig. 6) is underabundant with respect to iron, consistent with what is observed in the globular cluster NGC 6397 (James et al. 2004; Lind et al. 2011a) and in the field stars of similar metallicity (Burris et al. 2000; Fulbright 2000; Mashonkina \& Gehren 2001), but again, not inconsistent with what is observed in the higher metallicity stars of Sgr dSph (Sbordone et al. 2007). The average [Ba/Fe] of the two stars is nearly zero (Fig. 7), which is at variance with what is observed in NGC 6397 (James et al. 2004; Lind et al. 2011a), where [Ba/Fe] is $\sim-0.2$, although it should be stressed that in Galactic stars we see a large scatter in $[\mathrm{Ba} / \mathrm{Fe}]$ at this metallicity (Burris et al. 2000; Fulbright 2000; Mashonkina et al. 2003). Europium is strongly enhanced over iron in both stars, even more than what is found in NGC 6397 (James et al. 2004; Lind et al. 2011a), and we

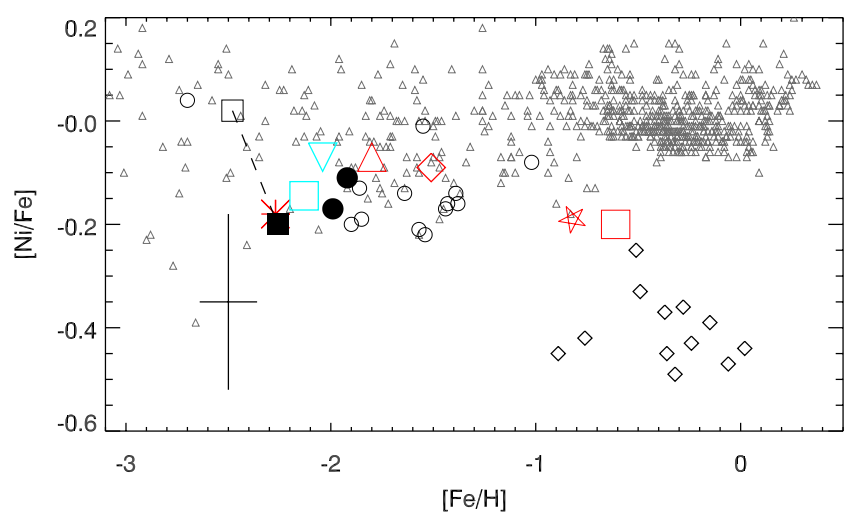

Fig. 5. $[\mathrm{Ni} / \mathrm{Fe}]$ vs. $[\mathrm{Fe} / \mathrm{H}]$ for the same samples, and using the same symbols as in Fig. 4.

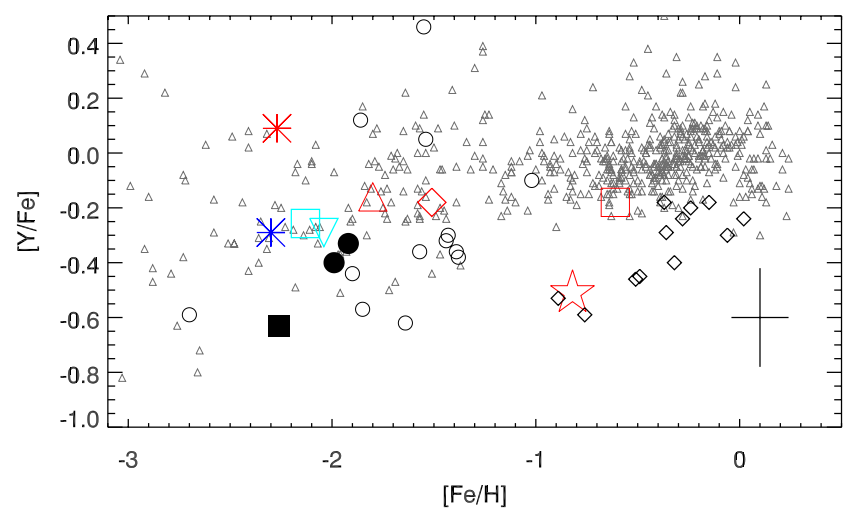

Fig. 6. $[\mathrm{Y} / \mathrm{Fe}]$ plotted against $[\mathrm{Fe} / \mathrm{H}]$. Symbols are the same as in Fig. 4 except for the large blue asterisk, which represents the average $\mathrm{Y}$ abundance in Ter 8 as measured by Mottini et al. (2008). Here, in Fig. 7, and in Fig. 8 only the filled point appears for NGC 5053 since both Ba and $\mathrm{Y}$ are always compared to Fe II.

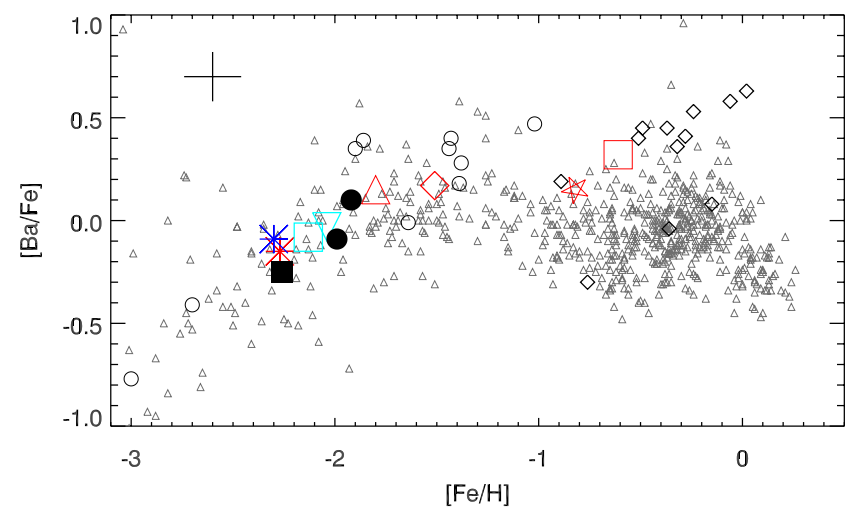

Fig. 7. $[\mathrm{Ba} / \mathrm{Fe}]$ plotted against $[\mathrm{Fe} / \mathrm{H}]$, symbols as in Fig. 6.

also observe a large scatter in $[\mathrm{Eu} / \mathrm{Fe}]$ among Galactic stars at this metallicity (Burris et al. 2000; Fulbright 2000; Mashonkina et al. 2003), while it has been found essentially at the solar value in the two solar metallicity Sgr dSph stars analyzed by Bonifacio et al. (2000).

\subsection{NGC 5053}

The single star analyzed in NGC 5053 shows a metallicity of $[\mathrm{Fe} I \mathrm{II} / \mathrm{H}]=-2.26 \pm 0.01$, in excellent agreement with the value 


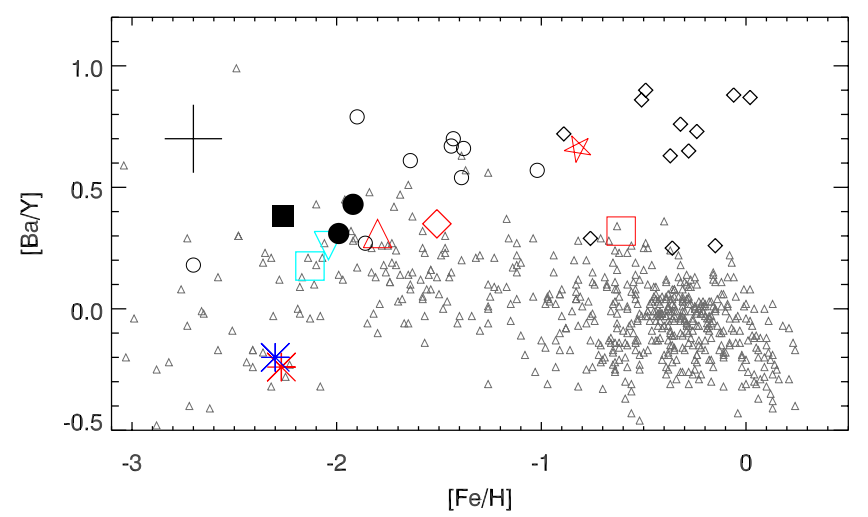

Fig. 8. $[\mathrm{Ba} / \mathrm{Y}]$ plotted against $[\mathrm{Fe} / \mathrm{H}]$, see Fig. 6 for the symbol legend.

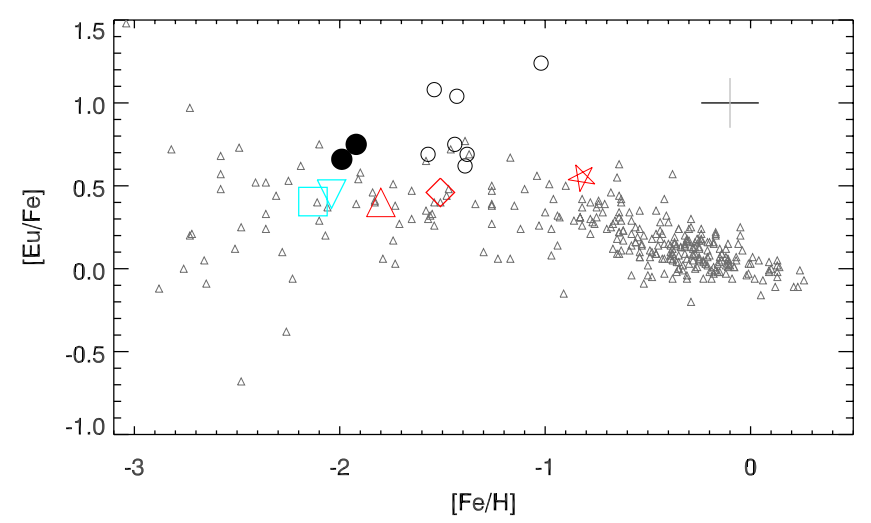

Fig. 9. $[\mathrm{Eu} / \mathrm{Fe}]$ plotted against $[\mathrm{Fe} / \mathrm{H}]$, see Fig. 4 for the symbol legend.

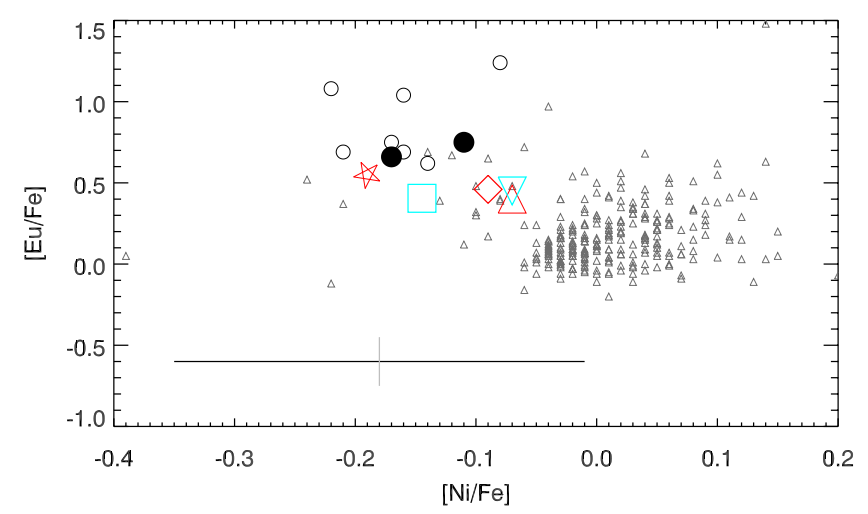

Fig. 10. $[\mathrm{Eu} / \mathrm{Fe}]$ plotted against $[\mathrm{Ni} / \mathrm{Fe}]$, see Fig. 4 for the symbol legend.

of -2.27 in Harris (1996), based in large part on the Ca triplet analysis of 11 stars by Geisler et al. (1995).

The choice of employing Fe II as the reference iron abundance and of assuming photometric atmosphere parameters has relevant effects on the $[\mathrm{X} / \mathrm{Fe}]$ abundance ratios listed in Table 2; since $[\mathrm{Fe} \mathrm{I} / \mathrm{H}]$ is 0.22 dex lower than $[\mathrm{Fe} \mathrm{II} / \mathrm{H}]$, if $[\mathrm{Fe} \mathrm{I} / \mathrm{H}]$ were used as reference, as is usually done for neutral species, all the neutral $[\mathrm{X} / \mathrm{Fe}]$ ratios would be $0.22 \mathrm{dex}$ higher. The choice of using Fe II as reference was motivated by the belief that Fe I is significantly affected by NLTE and 3D effects in this star. However, at the present time NLTE/3D corrections are only available for a handful of species, so that we generally do not know whether the same is true for other neutral species, and to what extent. If we assumed, for instance, that a given neutral species was affected in the same way as Fe I, for that species the ratio against

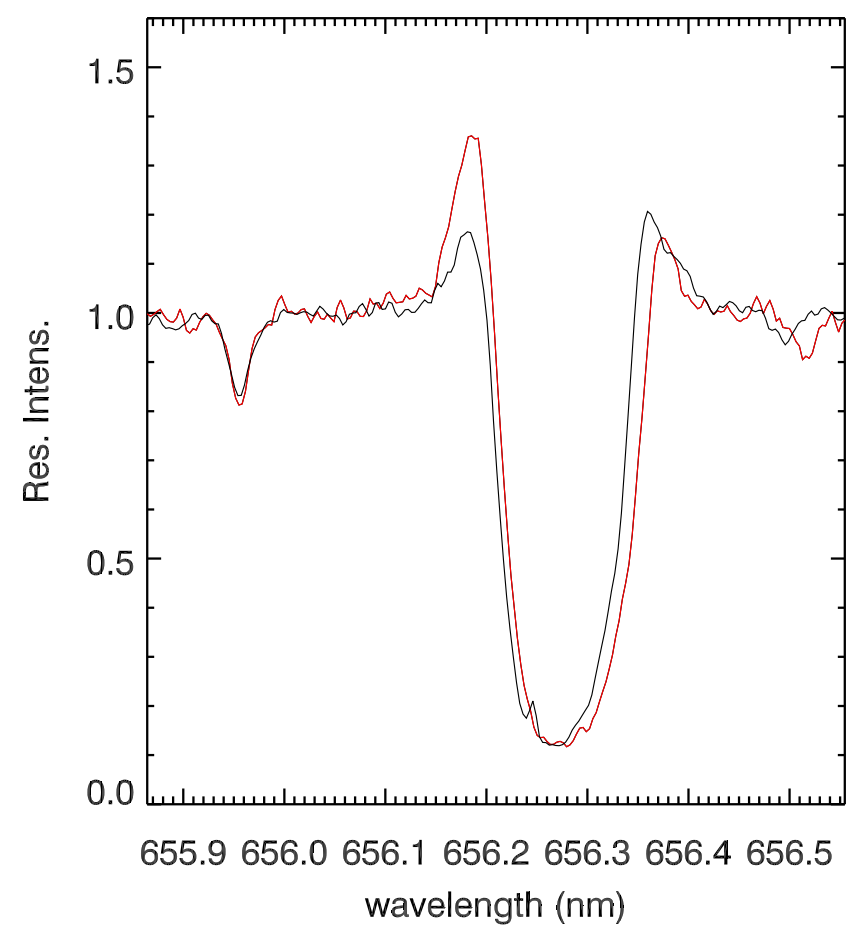

Fig. 11. $\mathrm{H} \alpha$ line in the spectra of the stars NGC 5634-2 (black) and NGC 5634-3 (red), normalized and shifted to zero velocity. The absorption line on the blue side is the Ti II $655.959 \mathrm{~nm}$.

Fe I would be the appropriate one, while the ratio against Fe II is the correct one if one assumes the species to be unaffected by NLTE/3D. As such, star NGC 5053-69 is difficult to plot in Figs. 4 and 5: to make the issue visible, we plotted the results for this star with a double symbol, the filled one indicating the abundance ratio as listed in Table 2, the open one plotting instead $[\mathrm{X} / \mathrm{Fe} \mathrm{I}]$ vs. [Fe I/H].

Sodium is measured in NGC 5053-69 only through the $568.8 \mathrm{~nm}$ line and delivers a fairly high abundance $([\mathrm{Na} / \mathrm{Fe} \mathrm{II}]=0.21)$, about 0.4 dex higher than expected for a first-generation star. The NLTE correction computed through INSPECT for this line (assuming $\log g=1.0$ ) is of -0.04 dex (in the sense of the NLTE-corrected abundance being lower). This would hint at the presence of multiple stellar generations in NGC 5053 as well.

While the present paper was undergoing the refereeing process, an independent analysis of NGC 5053 based on medium resolution spectra $(R \sim 13000)$ appeared as a preprint (Boberg et al. 2015). They observed star NGC 5053-69, (star 6 in their list). In spite of the lower resolution and limited spectral range, their derived atmospheric parameters are in remarkably good agreement with ours $\left(130 \mathrm{~K}\right.$ difference in $T_{\text {eff }},-0.05$ in $\log g$ and -0.07 dex in $[\mathrm{Fe} / \mathrm{H}])$. They manage to measure $[\mathrm{O} / \mathrm{Fe}]$ from the $[\mathrm{OI}] 630 \mathrm{~nm}$ line and find $[\mathrm{O} / \mathrm{Fe}]=-0.2$, which is consistent with their enhanced sodium $([\mathrm{Na} / \mathrm{Fe}]=0.6)$. Sodium is one of the most discrepant elements with respect to our analysis. We did not use the same lines: they used the $616.2 \mathrm{~nm}$ line, while we used the $568.2 \mathrm{~nm}$ and $568.8 \mathrm{~nm}$ lines. We also adopted different NLTE corrections; Boberg et al. (2015) used those of Gratton et al. (1999). The other element that is distinctly different between the two analyses is Ca. Boberg et al. (2015) derive $[\mathrm{Ca} / \mathrm{Fe}]=+0.49$, while we derive +0.10 . Considering that our $\mathrm{Ca}$ abundance relies on nine $\mathrm{Ca} \mathrm{I}$ lines and $[\mathrm{Ca} / \mathrm{Fe}]$ is in excellent agreement with $[\mathrm{Si} / \mathrm{Fe}]$ in this star (also derived from nine lines), we believe that our result is very robust. Given that no 
details on the lines used are given in the preprint, we cannot make a hypothesis about the reason of this discrepancy. With the two exceptions of $\mathrm{Na}$ and $\mathrm{Ca}$, the agreement on the abundance ratios for the other elements in common $(\mathrm{Ti}, \mathrm{Ni}, \mathrm{Ba})$ is excellent.

Strong underabundances of $\mathrm{Cr}$ and $\mathrm{Mn}$, with respect to iron, are observed in this star, in line with what is observed in Milky Way stars; in both cases this is likely due to the neglect of NLTE effects (Bonifacio et al. 2009; Bergemann \& Cescutti 2010; Bergemann \& Gehren 2008).

Both the neutron capture elements $\mathrm{Y}$ and $\mathrm{Ba}$ are found to be underabundant with respect to iron. For Y this is similar to what is observed in the more metal-rich stars of Sgr dSph (Sbordone et al. 2007). For Ba instead, all the giant stars observed by (Sbordone et al. 2007) are enhanced in Ba. Again, we would like to stress that for both elements a large scatter is observed in Galactic stars at these metallicities (Burris et al. 2000; Fulbright 2000; Mashonkina \& Gehren 2001; Mashonkina et al. 2003).

\subsection{Assessing the proposed association with Sgr dSph}

The association of NGC 5053 and NGC 5634 with Sgr dSph (Bellazzini et al. 2003b; Law \& Majewski 2010) has so far been exclusively based on their position and kinematic properties, and is thus probabilistic. One of the obvious reasons for exploring the chemistry of these clusters is thus to look for characteristics that may set them aside from the typical MW behavior at similar metallicities, but that are shared with known Sgr dSph populations. Such a chemical signature is rather dramatic at higher metallicity (Cohen 2004; Sbordone et al. 2007), but it becomes more and more difficult to discern as metallicity decreases, since Sgr dSph chemical evolution at low metallicities appears to closely match the values observed in the MW halo (e.g. McWilliam et al. 2013). We will now look in greater detail at some chemical markers that might be used to infer an association of NGC 5053 and NGC 5634 with Sgr dSph.

In Fig. 4 , the $[\mathrm{X} / \mathrm{Fe}]$ ratios for the three $\alpha$-elements $\mathrm{Mg}$, $\mathrm{Si}$, and $\mathrm{Ca}$, and the mixed- $\alpha$-Fe-peak Ti in NGC 5053 and NGC 5634 are compared with Sgr dSph main body populations, the MW halo, globular clusters associated with Sgr dSph, and globular clusters thought to have originally formed in the MW. Since the $[\alpha / \mathrm{Fe}]$ ratio in Sgr dSph closely follows the MW value below $[\mathrm{Fe} / \mathrm{H}] \sim-1.2$, we do not expect to see any odd behavior here. In fact, NGC 5634 behaves as expected. The one star analyzed in NGC 5053, on the other hand, displays strikingly low $[\mathrm{Ca} / \mathrm{Fe}]$ and $[\mathrm{Ti} / \mathrm{Fe}]$ when $\mathrm{Fe}$ II is employed as reference, but it would fall in line with all the other displayed populations if Fe I were used as reference.

Nickel (Fig. 5) is another element whose ratio to iron is characteristically low in metal-rich $\mathrm{Sgr} \mathrm{dSph}$ populations with respect to the MW. However, the Galactic distribution of [Ni/Fe] becomes more dispersed at lower metallicity. While NGC 5053 and NGC 5634, together with confirmed Sgr dSph system member clusters, remain toward the lower end of the MW field stars $[\mathrm{Ni} / \mathrm{Fe}]$ values, they are fully compatible with the abundances in NGC 5897 and NGC 6397.

Yttrium and barium abundances (see Figs. 6 and 7) as well as their ratio (Fig. 8) are also rather peculiar in the more metalrich Sgr dSph populations (see e.g. Fig. 12 in Carretta et al. 2014). Yttrium in NGC 5634 and, in particular, in NGC 5053, appears lower than in the MW halo population, and is coherent with both metal-rich and metal-poor Sgr dSph main body populations. Both NGC 5053 and NGC 5634 actually appear a little more yttrium-poor than the other known Sgr dSph clusters, with the exception of Palomar 12. A somewhat puzzling case here is Terzan 8 whose $[\mathrm{Y} / \mathrm{Fe}]$ ratio is derived in Mottini et al. (2008) and Carretta et al. (2014), but is reported on average as 0.4 dex lower in the former. The difference persists in the one star the two studies have in common where $\mathrm{Y}$ was measured. Since the Mottini et al. (2008) abundance is in better agreement with the general trend of Sgr dSph populations, we plot it as well (as a blue large asterisk) in Figs. 6 and 8. At any rate, again, NGC 5897 and NGC 6397 are not significantly removed from what is found in NGC 5634.

Barium is generally enhanced in the more metal-rich Sgr dSph populations, but it has been known for a while that a subpopulation exists of Ba-poor Sgr dSph stars (Fig. 5 in Sbordone et al. 2007, and Fig. 7 here), whose origin is unclear. The high $[\mathrm{Ba} / \mathrm{Fe}]$ ratio, and the connected high $[\mathrm{Ba} / \mathrm{Y}]$ ratio, are among the Sgr dSph chemical signatures that persist to the lowest metallicity, as shown in Fig. 8. However, even this chemical peculiarity disappears around $[\mathrm{Fe} / \mathrm{H}]=-2.0$, most likely as an effect of the diminishing relevance of s-process at low metallicities. Seen in this context, NGC 5053 and NGC 5634 nicely follow a general trend toward low $[\mathrm{Ba} / \mathrm{Fe}]$ at low metallicity, and higher-than-MW values at high metallicity, which is discernible among the Sgr dSph core populations (with the notable exception of the aforementioned low-Ba population), and the associated globular clusters. However, as said above, NGC 5053 and NGC 5634 Ba abundances are also compatible with MW values, and a similar picture is also drawn by the $[\mathrm{Ba} / \mathrm{Y}]$ ratio.

Finally, [Eu/Fe] in NGC 5634 appears to be somewhat higher than typical for MW stars of comparable metallicity and for NGC 5897 and NGC 6397. Europium also shows a higher abundance here than in any other Sgr dSph cluster with the exception of the much more metal-rich Pal 12.

It is interesting to couple the results for $\mathrm{Ni}$ and $\mathrm{Eu}$ (as we do in Fig. 10). Here, the contemporary low $\mathrm{Ni}$ and high Eu abundance observed both in the Sgr dSph metal-poor population and in NGC 5634 sets them apart from the MW field and, to a lesser degree, from NGC 5897 and NGC 6397. Among Sgr dSph clusters, the same locus is shared by Pal 12 only, which is much more metal-rich than NGC 5634, while more metalpoor Sgr dSph clusters appear to agree more with the abundances found in MW globulars. Although this result is intriguing, its significance is limited by the rather large error bar on $\mathrm{Ni}$ abundances.

\section{Conclusions}

We present detailed chemical abundance studies of the distant halo globular clusters NGC 5053 and NGC 5634 based on three luminous giant stars. The star NGC 5053-69 has a metallicity of $[\mathrm{Fe} \mathrm{II} / \mathrm{H}]=-2.26 \pm 0.10$, while the two stars analyzed in NGC 5634 have metallicities of $[\mathrm{Fe} \mathrm{I} / \mathrm{H}]=-1.99 \pm 0.07$ and $-1.92 \pm 0.08$ for NGC 5634-2 and NGC 5634-3, respectively (uncertainties representing internal line-to-line scattering).

Star NGC 5634-2 appears to be O-rich and Na-poor, while NGC 5634-3 is 0.4 dex richer in $\mathrm{Na}$, and 0.3 dex poorer in $\mathrm{O}$, thus indicating the presence in NGC 5634 of the Na/O anticorrelation, almost universally observed in globular clusters. The high Na abundance in star NGC 5053-69 is also not consistent with the value expected in a first-generation star, indicating in agreement with Boberg et al. (2015) - that NGC 5053 hosts multiple stellar populations as well.

On the basis of their kinematics, both clusters are strongly suspected to have formed in the Sagittarius dwarf spheroidal 
galaxy, and to have been subsequently stripped by tidal interaction with the Milky Way. Although the Sgr dSph has a very characteristic set of chemical abundances in its metal-rich population, its more metal-poor stars resemble closely a typical halo composition, making it hard to use chemical abundances to assess the origin of these two clusters in the Sgr dSph system. Hints in this sense exist: NGC 5053 has a remarkably low yttrium abundance, NGC 5634 a higher than usual europium content, a finding which, when coupled with its nickel abundance, makes it strikingly similar to metal-poor Sgr dSph populations, and more different from MW field and GC stars. More generally, both clusters appear to fall, chemically, closer to Sgr dSph populations than to halo populations. All these conclusions combined make an origin for both clusters (NGC 5634 in particular) in the Sgr dSph system an appealing possibility. However, none of them appears strong enough to firmly confirm or reject this attribution.

Acknowledgements. Support for L.S. and S.D. is provided by Chile's Ministry of Economy, Development, and Tourism's Millennium Science Initiative through grant IC120009, awarded to The Millennium Institute of Astrophysics, MAS. C.M.B. gratefully acknowledges the support provided by Fondecyt reg. No. 1150060. S.V. gratefully acknowledges the support provided by Fondecyt reg. No. 1130721. M.B. acknowledges financial support from PRIN MIUR 20102011, project "The Chemical and Dynamical Evolution of the Milky Way and Local Group Galaxies", prot. 2010LY5N2T. D.G. gratefully acknowledges support from the Chilean BASAL Centro de Excelencia en Astrofísica y Tecnologías Afines (CATA) grant PFB-06/2007. E.C. is grateful to the FONDATION MERAC for funding her fellowship. Based in part on data collected at Subaru Telescope and obtained from the SMOKA, which is operated by the Astronomy Data Center, National Astronomical Observatory of Japan. This research has made use of NASA's Astrophysics Data System, and of the VizieR catalogue access tool, CDS, Strasbourg, France

\section{References}

Abadi, M. G., Navarro, J. F., Steinmetz, M., \& Eke, V. R. 2003, ApJ, 597, 21

Alonso, A., Arribas, S., \& Martínez-Roger, C. 1999, A\&AS, 140, 261

Alonso, A., Arribas, S., \& Martínez-Roger, C. 2001, A\&A, 376, 1039

Anders, E., \& Grevesse, N. 1989, Geochim. Cosmochim. Acta, 53, 197

Bellazzini, M., Ferraro, F. R., \& Ibata, R. 2002, AJ, 124, 915

Bellazzini, M., Ibata, R., Ferraro, F. R., \& Testa, V. 2003a, A\&A, 405, 577

Bellazzini, M., Ferraro, F. R., \& Ibata, R. 2003b, AJ, 125, 188

Bergemann, M., \& Cescutti, G. 2010, A\&A, 522, A9

Bergemann, M., \& Gehren, T. 2008, A\&A, 492, 823

Bergemann, M., Lind, K., Collet, R., Magic, Z., \& Asplund, M. 2012, MNRAS, 427, 27

Bihain, G., Israelian, G., Rebolo, R., Bonifacio, P., \& Molaro, P. 2004, A\&A, 423, 777

Boberg, O. M., Friel, E. D., \& Vesperini, E. 2015, ApJ, 804, 109

Bonifacio, P. 2005, 13th Cambridge Workshop on Cool Stars, Stellar Systems and the Sun, 560, 3

Bonifacio, P., Hill, V., Molaro, P., et al. 2000, A\&A, 359, 663

Bonifacio, P., Sbordone, L., Marconi, G., Pasquini, L., \& Hill, V. 2004, A\&A, 414, 503

Bonifacio, P., Spite, M., Cayrel, R., et al. 2009, A\&A, 501, 519

Bonifacio, P., Caffau, E., \& Ludwig, H.-G. 2010, A\&A, 524, A96

Burris, D. L., Pilachowski, C. A., Armandroff, T. E., et al. 2000, ApJ, 544, 302

Caffau, E., Ludwig, H.-G., Steffen, M., Freytag, B., \& Bonifacio, P. 2011a,

Sol. Phys., 268, 255

Carraro, G., \& Bensby, T. 2009, MNRAS, 397, L106

Carraro, G., Zinn, R., \& Moni Bidin, C. 2007, A\&A, 466, 181

Carretta, E., Bragaglia, A., Gratton, R. G., et al. 2010, A\&A, 520, A95

Carretta, E., Bragaglia, A., Gratton, R. G., et al. 2014, A\&A, 561, A87

Castelli, F. 2005, Mem. Soc. Astron. It. Supp., 8, 25

Cayrel, R., Depagne, E., Spite, M., et al. 2004, A\&A, 416, 1117

Coelho, P., Barbuy, B., Meléndez, J., Schiavon, R. P., \& Castilho, B. V. 2005, A\&A, 443, 735

Cohen, J. G. 2004, AJ, 127, 1545

Dean, J. F., Warren, P. R., \& Cousins, A. W. J. 1978, MNRAS, 183, 569

de Boer, T. J. L., Belokurov, V., Beers, T. C., \& Lee, Y. S. 2014, MNRAS, 443, 658
Dekker, H., D’Odorico, S., Kaufer, A., Delabre, B., \& Kotzlowski, H. 2000, Proc. SPIE, 4008, 534

Font, A. S., Johnston, K. V., Bullock, J. S., \& Robertson, B. E. 2006, ApJ, 638, 585

Font, A. S., Benson, A. J., Bower, R. G., et al. 2011, MNRAS, 417, 1260

Fulbright, J. P. 2000, AJ, 120, 1841

Geisler, D., Piatti, A. E., Claria, J. J., \& Minniti, D. 1995, AJ, 109, 605

Geisler, D., Wallerstein, G., Smith, V. V., \& Casetti-Dinescu, D. I. 2007, PASP, 119,939

Gratton, R. G. 1989, A\&A, 208, 171

Gratton, R. G., Carretta, E., Eriksson, K., \& Gustafsson, B. 1999, A\&A, 350, 955

Gratton, R. G., Bonifacio, P., Bragaglia, A., et al. 2001, A\&A, 369, 87

Gratton, R. G., Carretta, E., \& Bragaglia, A. 2012, A\&ARv, 20, 50

Harris, W. E. 1996, AJ, 112, 1487 (2010 edition)

Hesser, J. E., Shawl, S. J., \& Meyer, J. E. 1986, PASP, 98, 403

Ibata, R. A., Gilmore, G., \& Irwin, M. J. 1994, Nature, 370, 194

James, G., François, P., Bonifacio, P., et al. 2004, A\&A, 427, 825

Koch, A., \& McWilliam, A. 2014, A\&A, 565, A23

Kurucz, R. L. 2005, Mem. Soc. Astron. It. Supp., 8, 14

Lake, G. 1989, AJ, 98, 1554

Lanfranchi, G. A., \& Matteucci, F. 2003, MNRAS, 345, 71

Lanfranchi, G. A., \& Matteucci, F. 2004, MNRAS, 351, 1338

Law, D. R., \& Majewski, S. R. 2010, ApJ, 718, 1128

Lind, K., Charbonnel, C., Decressin, T., et al. 2011a, A\&A, 527, A148

Lind, K., Asplund, M., Barklem, P. S., \& Belyaev, A. K. 2011b, A\&A, 528, A103

Lodders, K., Plame, H., \& Gail, H.-P. 2009, Landolt-Börnstein - Group VI Astronomy and Astrophysics Numerical Data and Functional Relationships in Science and Technology Volume 4B: Solar System, ed. J. E. Trümper, 4.4, 44

Majewski, S. R., Skrutskie, M. F., Weinberg, M. D., \& Ostheimer, J. C. 2003, ApJ, 599, 1082

Mashonkina, L., \& Gehren, T. 2001, A\&A, 376, 232

Mashonkina, L., Gehren, T., Travaglio, C., \& Borkova, T. 2003, A\&A, 397, 275

Mashonkina, L., Gehren, T., Shi, J.-R., Korn, A. J., \& Grupp, F. 2011, A\&A, 528, A87

Mateo, M. L. 1998, ARA\&A, 36, 435

Mayall, N. U. 1946, ApJ, 104, 290

McWilliam, A., Rich, R. M., \& Smecker-Hane, T. A. 2003a, ApJ, 592, L21

McWilliam, A., Rich, R. M., \& Smecker-Hane, T. A. 2003b, ApJ, 593, L145

McWilliam, A., Wallerstein, G., \& Mottini, M. 2013, ApJ, 778, 149

Mishenina, T. V., Kovtyukh, V. V., Soubiran, C., Travaglio, C., \& Busso, M. 2002, A\&A, 396, 189

Monaco, L., Bellazzini, M., Ferraro, F. R., \& Pancino, E. 2004, MNRAS, 353, 874

Monaco, L., Bellazzini, M., Bonifacio, P., et al. 2005, A\&A, 441, 141

Monaco, L., Saviane, I., Correnti, M., Bonifacio, P., \& Geisler, D. 2011, A\&A, $525, \mathrm{~A} 124$

Mottini, M., Wallerstein, G., \& McWilliam, A. 2008, AJ, 136, 614

Nemec, J. M. 2004, AJ, 127, 2185

Noguchi, K., Aoki, W., Kawanomoto, S., et al. 2002, PASJ, 54, 855

Peterson, R. C. 1985, ApJ, 297, 309

Pryor, C., \& Meylan, G. 1993, Structure and Dynamics of Globular Clusters, 50, 357

Reddy, B. E., Lambert, D. L., \& Allende Prieto, C. 2006, MNRAS, 367, 1329

Stetson, P. B. 2000, PASP, 112, 925

Sbordone, L. 2005, Mem. Soc. Astron. It. Supp., 8, 61

Sbordone, L., Bonifacio, P., Castelli, F., \& Kurucz, R. L. 2004, Mem. Soc. Astron. It. Supp., 5, 93

Sbordone, L., Bonifacio, P., Marconi, G., Buonanno, R., \& Zaggia, S. 2005, A\&A, 437, 905

Sbordone, L., Bonifacio, P., Buonanno, R., et al. 2007, A\&A, 465, 815

Sbordone, L., Salaris, M., Weiss, A., \& Cassisi, S. 2011, A\&A, 534, A9

Sbordone, L., Caffau, E., Bonifacio, P., \& Duffau, S. 2014, A\&A, 564, A109

Searle, L., \& Zinn, R. 1978, ApJ, 225, 357

Shetrone, M. D., Côté, P., \& Sargent, W. L. W. 2001, ApJ, 548, 592

Shetrone, M., Venn, K. A., Tolstoy, E., et al. 2003, AJ, 125, 684

Siegel, M. H., Dotter, A., Majewski, S. R., et al. 2007, ApJ, 667, L57

Tolstoy, E., Hill, V., \& Tosi, M. 2009, ARA\&A, 47, 371

Tonry, J., \& Davis, M. 1979, AJ, 84, 1511

van den Bergh, S. 1998, ApJ, 505, L127

van den Bergh, S. 2000, PASP, 112, 529

Venn, K. A., Irwin, M., Shetrone, M. D., et al. 2004, AJ, 128, 1177

Vladilo, G., Sbordone, L., \& Bonifacio, P. 2003, The Local Group as an Astrophysical Laboratory, 107

Vogt, S. S., Allen, S. L., Bigelow, B. C., et al. 1994, Proc. SPIE, 2198, 362

Page 12 is available in the electronic edition of the journal at http://www . aanda.org 


\section{Appendix A: Line-by-line MyGlsFOS fits}

MyGIsFOS operates by fitting a region around each relevant spectral line against a grid of synthetic spectra, and deriving the best-fitting abundance directly, rather than using the line equivalent width (EW), which MyGIsFOS computes, but mainly to estimate $V_{\text {turb }}$. While this makes MyGIsFOS arguably more robust against the effect of line blends, it makes it rather pointless to provide a line-by-line table of EWs, atomic data, and derived abundances, unless atomic data for all the features included in the fitted region are provided, which is quite cumbersome.

To allow verification and comparison of our results, we thus decided to provide the per-feature abundances as well as the actual observed and synthetic best-fitting profile for each fitted region. We describe here in detail how these results will be delivered, since we plan to maintain the same format for any future MyGIsFOS-based analysis.

The ultimate purpose of these comparisons is to ascertain whether abundance differences between works stem from any aspect of the line modeling, and assess the amount of the effect. We believe that providing the observed and synthetic profiles is particularly effective in this sense for a number of reasons:

- The reader can apply whatever abundance analysis technique he/she prefers to either the observed or the synthetic spectra. For instance, if EWs are employed, he/she can determine the EW of either profile, and derive the abundance with his/her choice of atomic data, atmosphere models, and so on. This would allow him/her to directly determine what abundance the chosen method would assign to our best-fitting synthetic, and to compare it with the one we derive.

- The reader can evaluate whether our choice of continuum placement corresponds or differs from the one he/she applied, and assess the broadening we applied to the syntheses, both looking at the feature of interest, and looking for any other useful feature (e.g. unblended Fe I lines, which are often used to set the broadening for lines needing synthesis).

- The reader can directly assess the goodness of our fit with whatever estimator he/she prefers.

- The reader gains access to the actual observed data we employed for every spectral region we used. Not every spectrum is available in public archives, and even fewer are available in reduced, and (possibly) coadded form.

An example of MyGIsFOS fit results is presented in Fig. A.1. To make the results easy to handle by Vizier, they were split into two tables:

- The features table contains basic data for each region successfully fitted in every star. This includes a code identifying the star (e.g. N5053_69), the ion the feature was used to measure, the starting and ending wavelength, the derived abundance, the EWs for the observed and best-fitting synthetic (determined by integration under the pseudo-continuum),

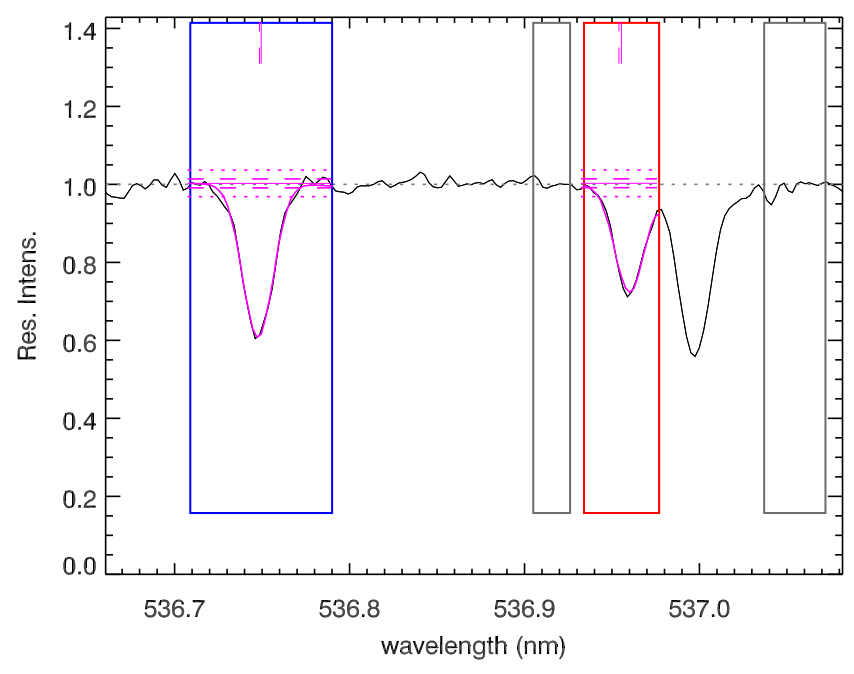

Fig. A.1. An example of MyGIsFOS fit for two features of star NGC 5634-2. The blue box corresponds to a Fe I feature, while the red box is a Co I feature. Gray boxes are pseudo-continuum estimation intervals. Observed pseudo-normalized spectrum is in black; magenta profiles are best-fitting profiles for each region. The black dotted horizontal line is the pseudo-continuum level, while the continuous thin magenta horizontal line represents the best-fit continuum for the feature. Around it, dashed and dotted horizontal magenta lines represent $1 \sigma$ and $3 \sigma$ intervals of the local noise $(S / N=88$ in this area). Vertical dashed and continuous lines mark the theoretical feature center, and the actual center after the best-fit, per-feature Doppler shift has been applied.

the local $\mathrm{S} / \mathrm{N}$, the small Doppler and continuum shift that MyGIsFOS allows in the fitting of each feature, flags to identify the Fe I features that were used in the $T_{\text {eff }}$ and $V_{\text {turb }}$ fitting process (the flags are not relevant for features not measuring Fe I), and finally a feature code formed of the element, ion, and central wavelength of the fitted range (e.g. 3000_481047). This last code is unique to each feature, and it can be used to retrieve the profile of the fit from the next table.

- The fits table contains all the fitted profiles for all the features for all the stars. Each line of the table corresponds to a specific pixel of a specific fit of a specific star, and contains the star identifying code, then the feature identifying code, followed by the wavelength, and the (pseudonormalized) synthetic and observed flux in that pixel. The user interested, for instance, in looking at the fit of the aforementioned $\mathrm{Zn}$ I feature in star NGC 5053-69, has simply to select from the table all the lines beginning with "N5053_69 3000_481047".

Both tables are available through CDS. To reproduce the fit as plotted in Fig. A.1, the synthetic flux must be divided by the continuum value provided in the features table. 